ARTICLE

Received 8 Sep 2015 | Accepted 15 Jun 2016 | Published 4 Aug 2016

DOI: $10.1038 /$ ncomms12237

OPEN

\title{
Normal stroma suppresses cancer cell proliferation via mechanosensitive regulation of JMJD1a-mediated transcription
}

Riina Kaukonen ${ }^{1}$, Anja Mai ${ }^{1}$, Maria Georgiadou${ }^{1}$, Markku Saari ${ }^{1}$, Nicola De Franceschi ${ }^{1}$, Timo Betz ${ }^{2}$, Harri Sihto ${ }^{3}$, Sami Ventelä1,4, Laura Elo1,5, Eija Jokitalo6, Jukka Westermarck1, Pirkko-Liisa Kellokumpu-Lehtinen7, Heikki Joensuu ${ }^{3,8}$, Reidar Grenman ${ }^{4} \&$ Johanna Ivaska1,9

Tissue homeostasis is dependent on the controlled localization of specific cell types and the correct composition of the extracellular stroma. While the role of the cancer stroma in tumour progression has been well characterized, the specific contribution of the matrix itself is unknown. Furthermore, the mechanisms enabling normal-not cancer-stroma to provide tumour-suppressive signals and act as an antitumorigenic barrier are poorly understood. Here we show that extracellular matrix (ECM) generated by normal fibroblasts (NFs) is softer than the CAF matrix, and its physical and structural features regulate cancer cell proliferation. We find that normal ECM triggers downregulation and nuclear exit of the histone demethylase JMJD1a resulting in the epigenetic growth restriction of carcinoma cells. Interestingly, JMJD1a positively regulates transcription of many target genes, including YAP/TAZ (WWTR1), and therefore gene expression in a stiffness-dependent manner. Thus, normal stromal restricts cancer cell proliferation through JMJD1a-dependent modulation of gene expression.

\footnotetext{
${ }^{1}$ Centre for Biotechnology, University of Turku, 20520 Turku, Finland. ${ }^{2}$ Institute of Cell Biology, Center for Molecular Biology of Inflammation, 48149 Muenster, Germany. ${ }^{3}$ Laboratory of Molecular Oncology, Translational Cancer Biology Program, University of Helsinki, 00290 Helsinki, Finland. ${ }^{4}$ Department of Otorhinolaryngology, Head and Neck Surgery, Turku University and Turku University Hospital, 20521 Turku, Finland. ${ }^{5}$ Department of Mathematics and Statistics, University of Turku, 20520, Turku, Finland. ${ }^{6}$ Institute of Biotechnology, Electron Microscopy Unit University of Helsinki, 00014 Helsinki, Finland. ${ }^{7}$ Department of Oncology, Tampere University Hospital, 36280 Tampere, Finland. ${ }^{8}$ Department of Oncology, Helsinki University Central Hospital, 00029 Helsinki, Finland. ${ }^{9}$ Department of Biochemistry and Food Chemistry, University of Turku, 20520 Turku, Finland. Correspondence and requests for materials should be addressed to J.I. (email: johanna.ivaska@utu.fi).
} 
n normal tissue, different cell types are spatially confined. For example, the normal epithelium is separated from the underlying stromal extracellular matrix (ECM) by the basement membrane, and thus they are not in a direct contact with each other. The stromal ECM is produced by the fibroblasts and serves as an important regulator of tissue homeostasis ${ }^{1}$. In contrast, invasive carcinomas contain a complex mixture of tumour cells and stromal components where the cancer cells interact with the altered ECM and are also embedded in it. Changes in the matrix stiffness as well as transition between twodimensional and three-dimensional matrix contacts influence cell proliferation profoundly ${ }^{2-5}$. Thus, cancer progression involves not only genetic alterations of cancer cells but also changes in the tumour microenvironment ${ }^{6}$. The role of the stroma as a potent antitumorigenic barrier has already been elucidated over two decades ago ${ }^{7,8}$. The exposure of carcinoma cells to normal basement membrane-like components has the capacity to revert breast cancer cells to a near-normal phenotype ${ }^{9}$. Furthermore, in coculture systems, normal fibroblasts (NFs) can inhibit the growth of certain cancer cells ${ }^{10,11}$, while a population of transformed cancer-associated fibroblasts (CAFs) can reverse the growth-inhibiting effect of $\mathrm{NFs}^{12}$. Thus, cross-talk between epithelial cells and fibroblasts is a critical feature of cancer progression. However, the specific contribution of the matrix itself has not been addressed in detail.

Tumour stromata are characterized by increased tissue stiffness and altered matrix architecture that favour proliferation, metastasis and drug resistance via aberrant mechanosignalling ${ }^{13-15}$. Stiffness also activates the main mediators of mechanotransduction: transcription factors YAP (Yes-associated protein) and TAZ (transcriptional coactivator with PDZ-binding motif) ${ }^{16}$. Epigenetic regulation is also implicated in cancer progression as it profoundly regulates the transcription profile and phenotype of cells. Many lines of evidence suggest that demethylation of repressive histone methylation marks, such as histone $\mathrm{H} 3$ lysine 9 (H3K9), from the gene promoters by histone demethylases is a prerequisite for transcriptional activation ${ }^{17-19}$. JMJD1A (KDM3A) demethylates monomethyl and dimethyl histone $\mathrm{H} 3 \mathrm{~K} 9$ in vitro and in vivo and has been implicated as a positive regulator of transcription of several growth-promoting genes ${ }^{17-19}$.

While the mechanisms whereby cancer stroma and CAFs contribute to tumour progression are being actively investigated, much less is known about how the normal stroma exerts tumoursuppressive signals to control tissue homeostasis. Furthermore, the role of epigenetic regulators in the ability of the cells to respond to the stiffness of the tumour microenvironment is not known. To investigate this, we compared the ability of matrices generated by NFs or CAFs from the same patient as well as matrices from immortalized NFs to influence cancer cell proliferation and gene expression. We find that the matrix generated by NFs, but not CAF matrix, profoundly inhibits cancer cell proliferation through mechanosensitive downregulation of the histone demethylase enzyme JMJD1a.

\section{Results}

Normal matrix inhibits cancer cell proliferation. Fibroblasts produce in vitro a robust cell-derived matrix (CDM) that recapitulates many features of the architecture and composition of in vivo $\mathrm{ECM}^{20}$. To investigate the potential effect of matrix on cancer cell proliferation, we generated matrices from telomerase-immortalized NFs (TIFFs) (Fig. 1a) and tested their effectiveness on two highly proliferative and widely studied cancer cell lines, namely cervical cancer HeLa and breast cancer MDA-MB-231 cells. Remarkably, both of these cell lines were significantly growth-inhibited (Fig. 1b) by the normal matrix compared with standard growth conditions on plastic. The growth-restrictive properties of CDM were only observed with the intact $\mathrm{CDM}$ as matrix proteins such as fibronectin or collagen I, or solubilized and re-plated CDM did not inhibit MDA-MB-231 cell proliferation (Supplementary Fig. 1a,b). Soluble factors were not implicated either because culturing the MDA-MB-231 cells in conditioned TIFF medium did not influence proliferation (Supplementary Fig. 1c). Thus, only the architecturally intact CDM possessed growth-inhibitory properties.

When investigating matrix-induced effects in more detail we, rather unexpectedly, observed that growth inhibition induced by TIFF CDM was maintained in cancer cells following detachment from the matrix by trypsinization and replating on plastic (Fig. 1c). Even though the matrix-exposed cells were returned to plastic in full serum-containing medium, both MDA-MB-231 and HeLa cells continued to proliferate significantly slower than the same cancer cell lines cultured continuously on plastic. Thus, exposure of cancer cells to CDM from NFs is not only growth-inhibitory but has the potential to revert malignant cancer cell proliferation in a sustained manner.

We observed that cancer cells grown on normal TIFF-derived CDM had significantly altered cell morphology compared with cells on plastic (Fig. 1d and Supplementary Fig. 1d). This was interesting as mechanical cues and environmental stiffness are known to affect the cytoskeleton and nuclear functions including chromatin condensation and global epigenetic status of a cell ${ }^{21-25}$, and therefore changes in cell morphology and gene expression could explain the matrix-dependent reversion in the cancer cell phenotype.

Matrix induces gene expression changes. We hypothesized that exposure to CDM could induce changes in epigenetic modifiers, hence suppressing cancer cell growth in a sustained manner. To investigate this possibility, we performed Illumina whole-genome transcription analysis in MDA-MB-231 and HeLa cells harvested directly from TIFF CDMs after 6 days (CDM), following detachment from CDMs and replating on plastic for 5 days (CDM to plastic) or in cells grown continuously on plastic (Fig. 1e). As CDM induced sustained growth inhibition in both cell lines, we focused our attention on common transcriptional alterations of epigenetic enzymes. A single well-characterized histone demethylase JMJD1a was significantly downregulated in both cell lines, suggesting that this gene might be linked to the sustained phenotypic alterations triggered by the CDM in both cancer cell lines. Among the other significantly altered genes were signalling proteins SORBS2 and $P D E 7 B$, which were downregulated in both cell lines and in both conditions (CDM and CDM to plastic; Fig. If and Supplementary Data 1). In addition, expression of 27 genes on CDM and 150 genes following CDM detachment were altered significantly in both cell lines. Several matrix-modifying proteins (MMP3, PLAUR1 and COL1A2) were upregulated on the matrix in both cell lines, while many genes involved in regulating cholesterol synthesis (IDI1, ACAT1 and HMGCS1) were downregulated on the matrix. Following matrix detachment, several hypoxia-related genes were downregulated (JMJD1A (KDM3A), ALDOC, DDIT4, GDF15, $A N G$ and $M U C 1)$ even though the rather thin CDM layer is unlikely to restrict oxygen diffusion in the in vitro cultures ${ }^{26}$. In addition, MYC-target genes (FAPB5 and NME1) were upregulated following CDM detachment and replating on plastic. Since the mechanism of sustained growth inhibition was of primary interest, we focused specifically on the epigenetic modifier enzyme JMJD1a that was downregulated following matrix detachment in both cell lines (Fig. 1f). 
a

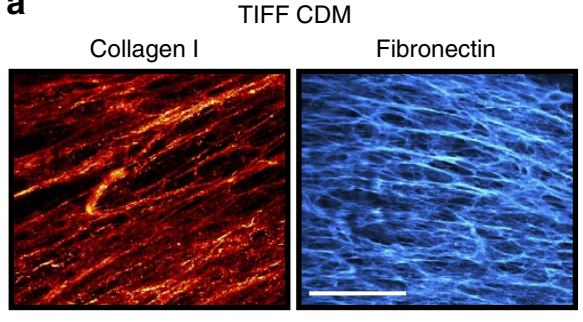

b

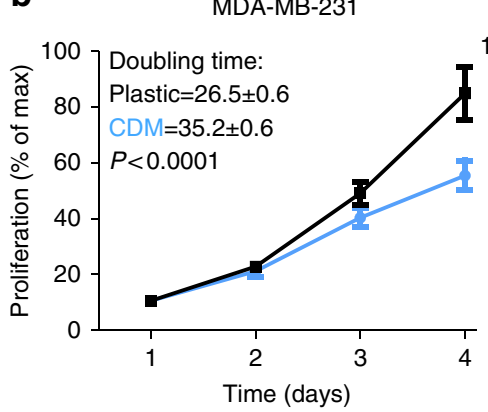

HeLa

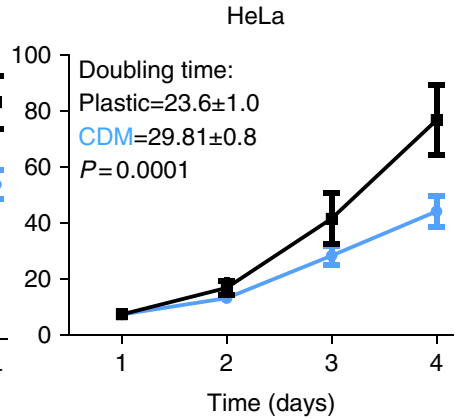

C
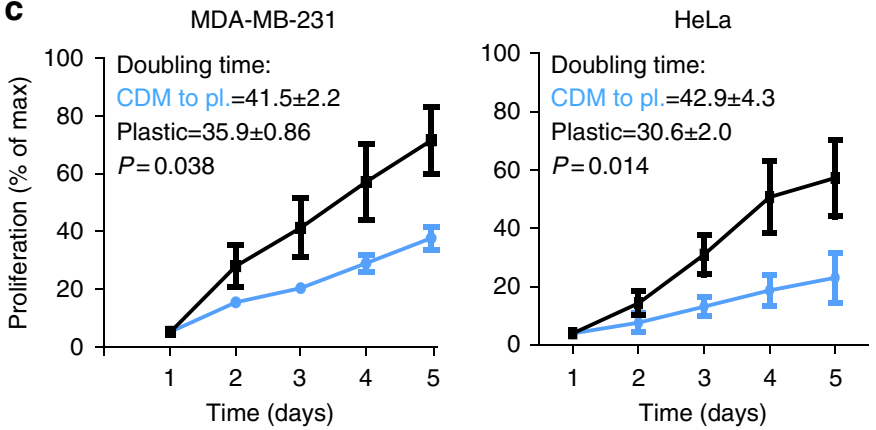

d
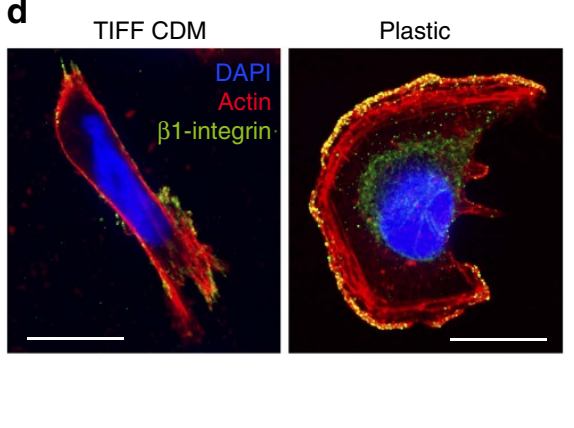

e

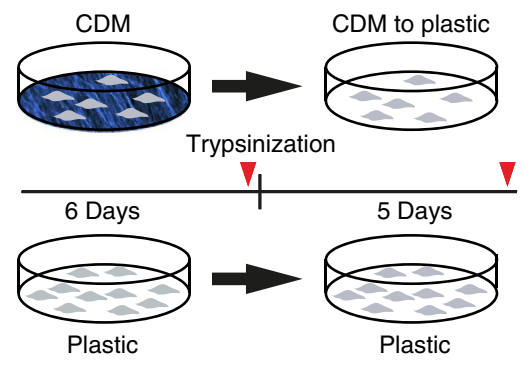

f CDM CDM to plastic

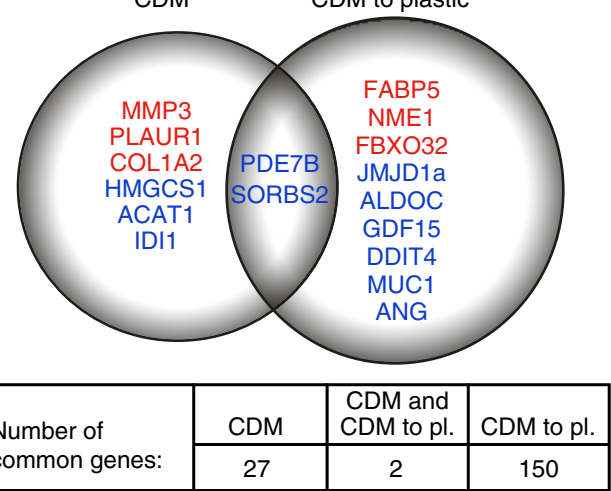

Figure 1 | Fibroblast-derived CDM induces sustained growth inhibition of cancer cells. (a) Collagen I and fibronectin staining of CDM generated by NFs (TIFFs). Scale bar, $20 \mu \mathrm{m}$. (b) Proliferation of MDA-MB-231 and HeLa cells plated on TIFF CDM or on plastic in full medium for the indicated times. $n(\mathrm{HeLa})=10, n(\mathrm{MDA}-\mathrm{MB}-231)=8$. (c) Proliferation of MDA-MB-231 and HeLa cells after detachment from TIFF matrices (6 days on matrix before detachment) and replating on plastic in full medium for the indicated times $n(\mathrm{HeLa})=7, n(\mathrm{MDA}-\mathrm{MB}-231)=10$. (d) Representative images of MDA-MB-231 cell morphology on CDM and plastic. Shown are maximum intensity projections of confocal images. Scale bar, $10 \mu \mathrm{m}$. (e) Schematic representation of the experimental set-up. Red arrows indicate the time points of sample collection for Illumina gene expression analysis. (f) Common gene expression changes in MDA-MB-231 and HeLa cells on CDM and 5 days after CDM detachment (CDM to plastic (pl.)). The numbers of commonly regulated genes (up- or downregulated) in both cell lines are indicated in the table. Upregulated genes are marked with red and downregulated genes with blue. All data are mean \pm s.e.m. Unpaired $t$-test was used for statistical analyses.

Normal matrix restrains JMJD1a expression. JMJD1a is a H3K9-specific demethylase that has been linked to several biological processes including growth, development and reprogramming ${ }^{17,27-29}$. Loss of JMJD1a decreases gene expression and increases the inhibitory $\mathrm{H} 3 \mathrm{~K} 9 \mathrm{me} 2$ modifications in the promoter regions of multiple genes ${ }^{19}$. We validated that JMJD1a was downregulated in cancer cells following detachment from CDM both on the protein (Fig. 2a,b) and on mRNA levels (Fig. 2c). Interestingly, JMJD1A was downregulated already in cells grown on CDM at the protein level (Fig. 2d,e), while the mRNA levels of JMJD1a were not changed (Fig. 2f). This correlated with reduced stability of the JMJD1a protein on CDM when compared with plastic (Fig. 2g), indicating that CDM-induced repression of JMJD1a first on the protein level and subsequently on mRNA level correlates with the CDM-induced growth inhibition of cancer cells when compared with cells grown on plastic (Fig. 1b,c). Silencing of JMJD1a recapitulated the growth inhibition in plastic-cultured cancer cells and induced a flat quiescent-looking morphology (Fig. 2h and Supplementary Fig. 1e-f). Conversely, proliferation was enhanced in cells with high JMJD1a-GFP levels when compared with high green fluorescent protein (GFP)-expressing cells on plastic (Fig. $2 \mathrm{i}$ and Supplementary Fig. 1g). Importantly, forced expression of JMJD1a was sufficient to rescue the CDM-induced growth inhibition on TIFF-derived CDM (Fig. 2j). Furthermore, the growth of MDA-MB-231 (breast cancer) cells in an in vivo chicken embryo chorioallantoic membrane (CAM) assay as well as orthotopic tumour growth in mice were significantly reduced 
a

MDA-MB-231

CDM to

$M_{\mathrm{r}}(\mathrm{K})$ plastic Plastic

$150-\approx$ JMJD1a

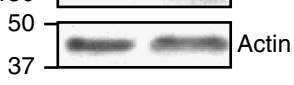

HeLa

CDM to

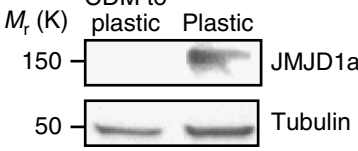

d

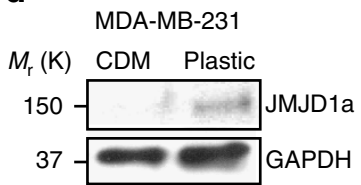

HeLa

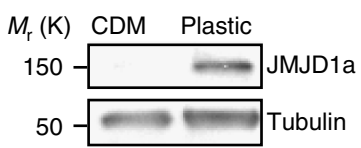

h

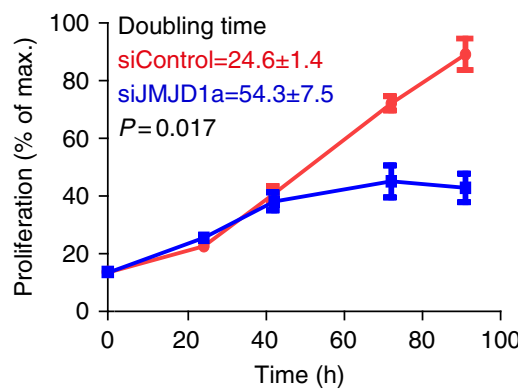

k

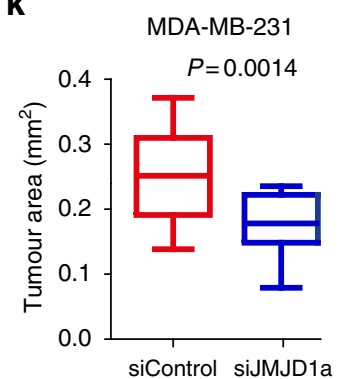

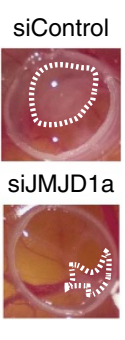

b

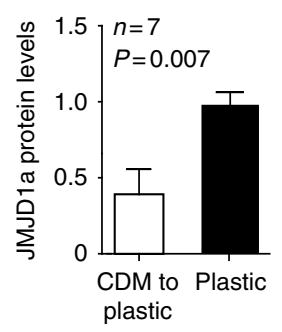

e

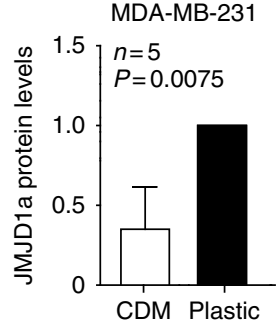

C

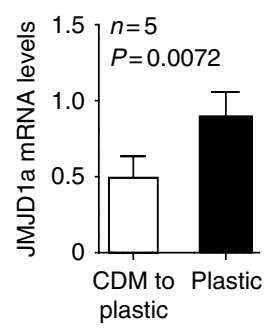

f

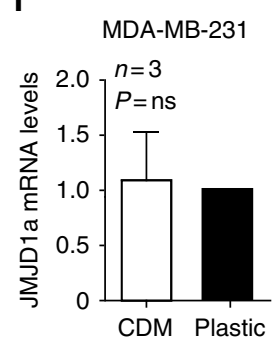

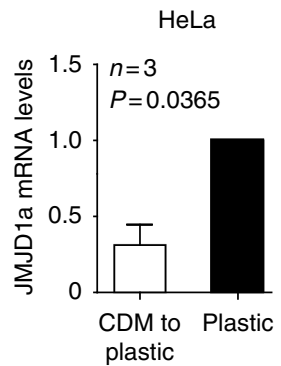

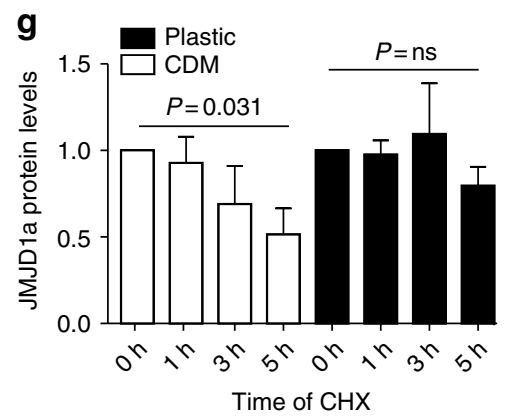

j Proliferation on CDM
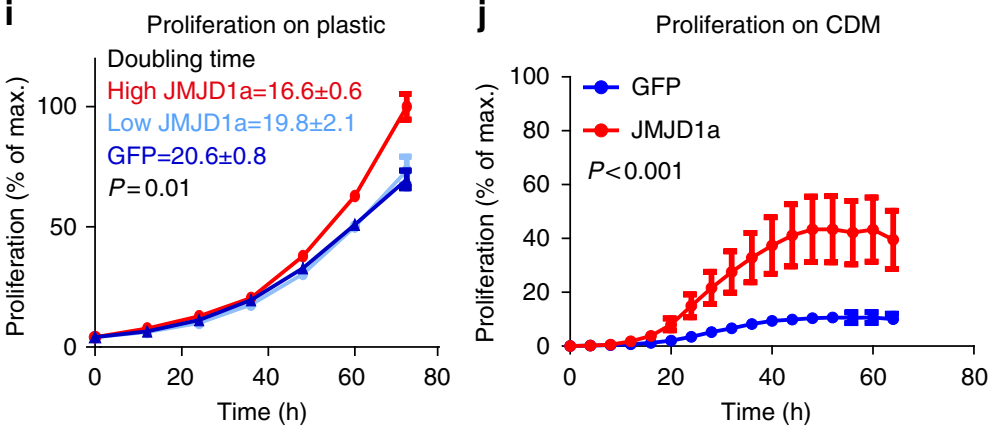

I

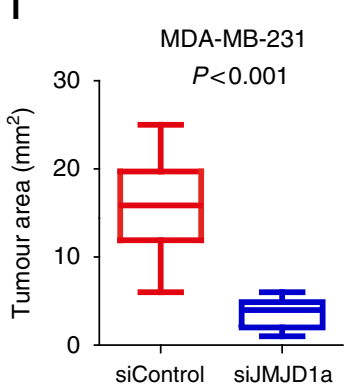

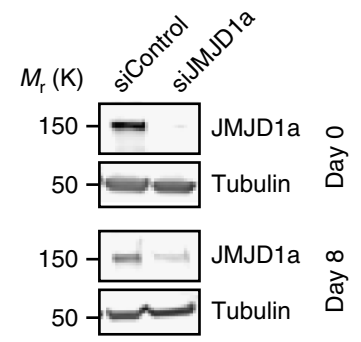

๙ั

Figure 2 | Normal ECM restrains JMJD1a expression, cell proliferation and tumour growth. (a,b) Western blot (a) and quantification (b) of JMJD1a protein levels normalized to loading control. (c) JMJD1a mRNA expression (qRT-PCR) relative to GAPDH mRNA in MDA-MB-231 and HeLa cells after matrix detachment ( 6 days on TIFF CDM and 5 days on plastic; CDM to plastic) and on plastic. (d,e) Western blot (d) and quantification of JMJD1a protein levels (e) normalized to loading control. (f) JMJDla mRNA expression (qRT-PCR) relative to GAPDH mRNA (f) in the indicated cells plated on either TIFF CDM or on plastic for 4 days. (g) Western blot quantification showing JMJD1a stability on CDM and plastic $24 \mathrm{~h}$ after plating. Time of the cycloheximide $(\mathrm{CHX})$ treatment is indicated and $P$ values are calculated between 0 and $5 \mathrm{~h}$. Paired t-test was used for statistical analysis, $n=3$. (h) Proliferation of MDA-MB-231 cells upon JMJD1a silencing on plastic, $n=3$. (i) Proliferation of JMJD1a-GFP or GFP-overexpressing MDA-MB-231 cells on plastic. Cells were sorted by FACS (JMJD1a: high and low; GFP: high), $n=3$. (j) Proliferation of GFP control and JMJD1a-GFP-overexpressing MDA-MB-231 cells on TIFFderived CDM. $n$ (GFP) $=11$ CDMs and $n$ (JMJD1a-GFP) $=12$ CDMs. Two-way analysis of variance (ANOVA) was used to calculate the $P$ value. Data are mean \pm s.e.m. (k) Control or JMJD1a siRNA-transfected MDA-MB-231 cells $\left(1 \times 10^{6}\right)$ were implanted on CAM membranes inside a plastic ring to analyse tumour growth in vivo for 3 days. Shown are quantified tumour areas from three individual experiments $n($ siControl $)=25, n(s i J M J D 1 a)=23$ eggs.

(I) Orthotopic tumour growth assay. Control or JMJD1a siRNA-transfected MDA-MB-231 cells $\left(2 \times 10^{6}\right)$ were injected into the fat pad of nude mice $(n=19)$ and tumour growth was measured 8 days after injection. Western blot in showing the silencing efficacy of JMJD1a siRNA on the day of the injection (Day 0) and at the end of the experiment (Day 8). Shown are mean \pm s.d. and (g-i) mean \pm s.e.m. Paired $t$-test was used for statistical analyses in b-h and non-paired $t$-test in $\mathbf{j}, \mathbf{k}$. 
upon silencing of JMJD1a (Fig. 2k,l). Thus, JMJD1a is downregulated following CDM exposure of cells and is a potent regulator of cancer cell proliferation in vitro and in vivo.

NFs and CAFs generate architecturally distinct CDM. To test the ability of stromal ECM to influence cancer cells in a more clinically relevant model, we isolated CAFs from the tumour stroma of three head and neck squamous cell carcinoma (HNSCC) patients (Supplementary Fig. 2a) and NFs from an unaffected area of the same patients. CAFs were identified by the high expression of CAF marker smooth muscle actin- $\alpha$ ( $\alpha$-SMA), which was low or absent in the NFs and the TIFFs (Fig. 3a and Supplementary Fig. 2b). RNA-sequencing demonstrated that all three CAFs and NFs clustered together (Supplementary Fig. 2c,d), which indicated that CAFs derived from different patients were more similar to other CAFs than to their corresponding NFs and all the NFs resembled each other (Supplementary Fig. 2c,d). However, all NF and CAF cell lines expressed genes, which are typically highly expressed by fibroblasts, such as Vimentin, Fibronectin 1 and several different collagens validating that they are fibroblasts (Supplementary Fig. 2e). CAFs had elevated levels of YAP and TAZ (Fig. 3b-d) compared with NFs, and they were also more able to contract collagen gels (Supplementary Fig. 2f), in line with a previous report on the role of YAP and contractility in the CAF phenotype ${ }^{30}$. In addition, we observed a significant upregulation of $\beta 1$-integrin, which has also been connected to contractility and mechanosignalling (Supplementary Fig. 2g,h).

We analysed the CDM produced by the different fibroblasts using immunofluorescence and scanning electron microscopy (SEM). Immunofluorescence staining revealed that similarly to TIFF CDM (Fig. 1a) the matrix bundles in NF CDM were more sparse compared with the corresponding CAF matrix, which displayed a denser and more uniform collagen and fibronectin staining (Fig. 3e and Supplementary Fig. 3a,b). Furthermore, based on SEM analysis, the NF and TIFF CDMs had more uniform and aligned structures compared with CAF-derived matrices (Fig. 3f). According to the RNA-sequencing data, the mRNA expression of different types of collagen or fibronectin was not changed, suggesting that matrix assembly and/or turnover, rather than production, results in distinct CDM architecture between NF and CAF CDM. However, even though we could not find significant differences on mRNA levels of matrix-related genes or in cell adhesion to solubilized and re-plated NF and CAF CDM (Supplementary Fig. 3c), the NF and CAF CDMs may differ in their protein composition as suggested earlier by others ${ }^{31}$.

These data show that CDMs generated by NFs and CAFs differ and that CAF-derived matrices from different patients share similar features that are distinct from matrices made by NFs from the same individual.

CAF matrix lacks growth-inhibitory properties. To test the ability of the patient-derived stromal ECM to influence the proliferation of cancer cells, we cultured MDA-MB-231 and HeLa cells on CDMs derived from either NFs or CAFs. Interestingly, NF CDM was significantly growth-inhibitory compared with CDM generated by CAFs from the same patient (Fig. 3g), and the same was observed when comparing TIFF and CAF CDM (Supplementary Fig. 3d). Importantly, also the growth of the patient-derived primary squamous cell carcinoma (SCC) cells was inhibited by the NF CDM matrix compared with the CAF CDM (Supplementary Fig. 3e), demonstrating that the growthrestrictive ability of NF CDM is widely applicable to different carcinomas. Similarly to the TIFF CDM, the growth restriction was specifically due to the matrix and not soluble factors as coculture of SCC or MDA-MB-231 cells with NFs or CAFs separated by a filter or conditioned medium from NFs or CAFs had no effect on proliferation (Supplementary Fig. 3f-h). In line with results obtained in breast cancer cells, JMJD1a silencing was sufficient to inhibit the proliferation of patient-derived SCC cells (Supplementary Fig. 3i). Concurrent with the ability of NF-derived CDM to downregulate JMJD1a levels, cancer cells on plastic and CAF CDM expressed high levels of JMJD1a, whereas JMJD1a was downregulated on normal CDMs (TIFF and NF; Fig. 3h), further validating the ability of normal CDM to restrict proliferation by JMJD1a downregulation.

JMJD1a levels correlate with activated stroma within tumours. The data above demonstrate that NF CDM downregulates and CAF CDM supports levels of JMJD1a. We found histologic features in human breast cancer and HNSCC tumours that are compatible with our experimental model, suggesting that the CAF matrix supports JMJD1a expression. We observed that JMJD1a expression coincides with the presence of $\alpha$-SMA-positive stromal cells, which are characteristic for the tumour microenvironment ${ }^{32,33}$. Staining of 10 normal breast tissue samples, 28 primary breast cancer sections and 7 lymph node metastasis revealed that in normal breast tissues JMJD1a expression was low or absent and $\alpha$-SMA was restricted to the basal mammary epithelial cells (Fig. 4a). In contrast, 27/28 of the breast cancers were JMJD1a-positive (low: 57\%; intermediate: 29\%; high: $11 \%$ ) and $27 / 28$ of the tumours had $\alpha$-SMA-positive stroma (Fig. 4a). In addition, all metastases were positively stained for JMJD1a and $\alpha$-SMA. In HNSCC patient samples, $10 / 14$ of the cancers were JMJD1a- and $\alpha$-SMA-positive (JMJD1a low: 50\%; JMJD1a high: 21\%). All HNSCC samples that were highly JMJD1a-positive also exhibited intense $\alpha$-SMA expression in the stroma (Fig. 4b). These analyses suggest that JMJD1a is expressed in breast and HNSCC carcinomas and correlates with the presence of $\alpha$-SMA-positive stroma in patients. Since increased matrix stiffness has been linked to cancerassociated stromal alterations and cell proliferation, we measured the stiffness of the patient-derived fibroblast-generated CDMs and TIFF CDM using atomic force microscopy (AFM). High indentation forces of up to $30 \mathrm{nN}$ were applied. Pairwise comparison of matrices generated from fibroblasts from the same patient demonstrated that CAF-derived matrices were significantly stiffer than the normal matrix (Fig. 4c). In addition, stiffness of the TIFF CDM was similar to the NF CDMs, in line with the similar growth-inhibitory properties of the matrices (Fig. 4d). These stiffness values for NF-generated CDMs and the higher range of stiffness in the CAF CDM are highly consistent with earlier measurements on CAF-contracted collagen gels ${ }^{30}$ and differences in tissue stiffness observed in normal breast tissue and cancer ${ }^{34}$.

JMJD1a levels and localization are regulated by stiffness. The requirement for intact ECM and the fact that normal CDM was less stiff compared with the CAF CDM suggested that matrix stiffness, in addition to matrix architecture and possibly composition, could be involved in the ability of the normal matrix to inhibit cancer cell proliferation. To test this in a controlled manner, cancer cells were grown on collagen I-coated hydrogels of varying stiffness. MDA-MB-231, HeLa and patient-derived SCC cells proliferated significantly more on stiffer supports (Supplementary Fig. 4a,b), suggesting that the lower stiffness of NF CDM is likely to contribute to its growth-restrictive properties.

JMJD1a has thus far been reported to localize to the nucleus $19,27,35$ in line with its function as a histone demethylase. However, nuclear fractionation analyses revealed that JMJD1a localizes both to the nucleus and to the cytoplasm in 

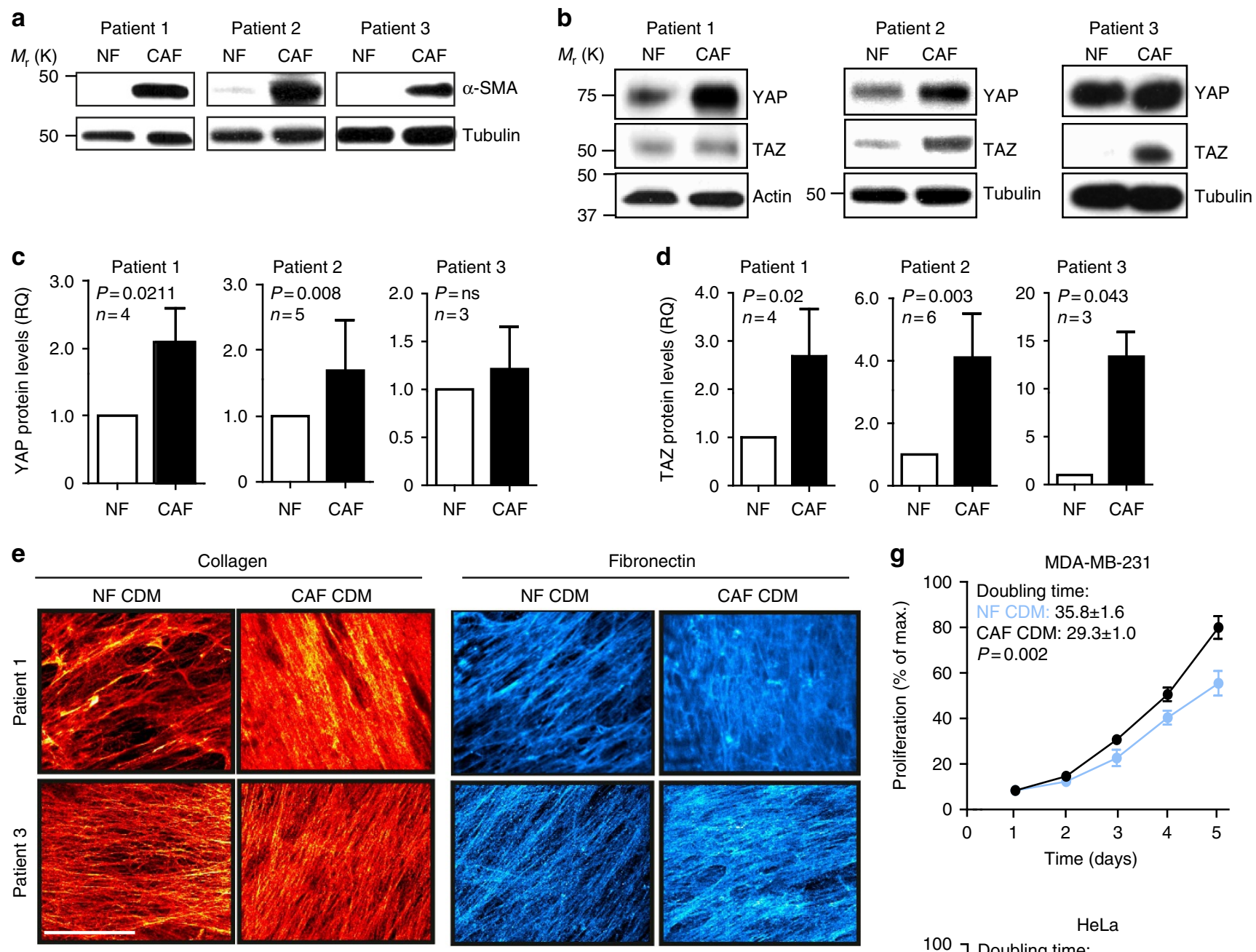

f
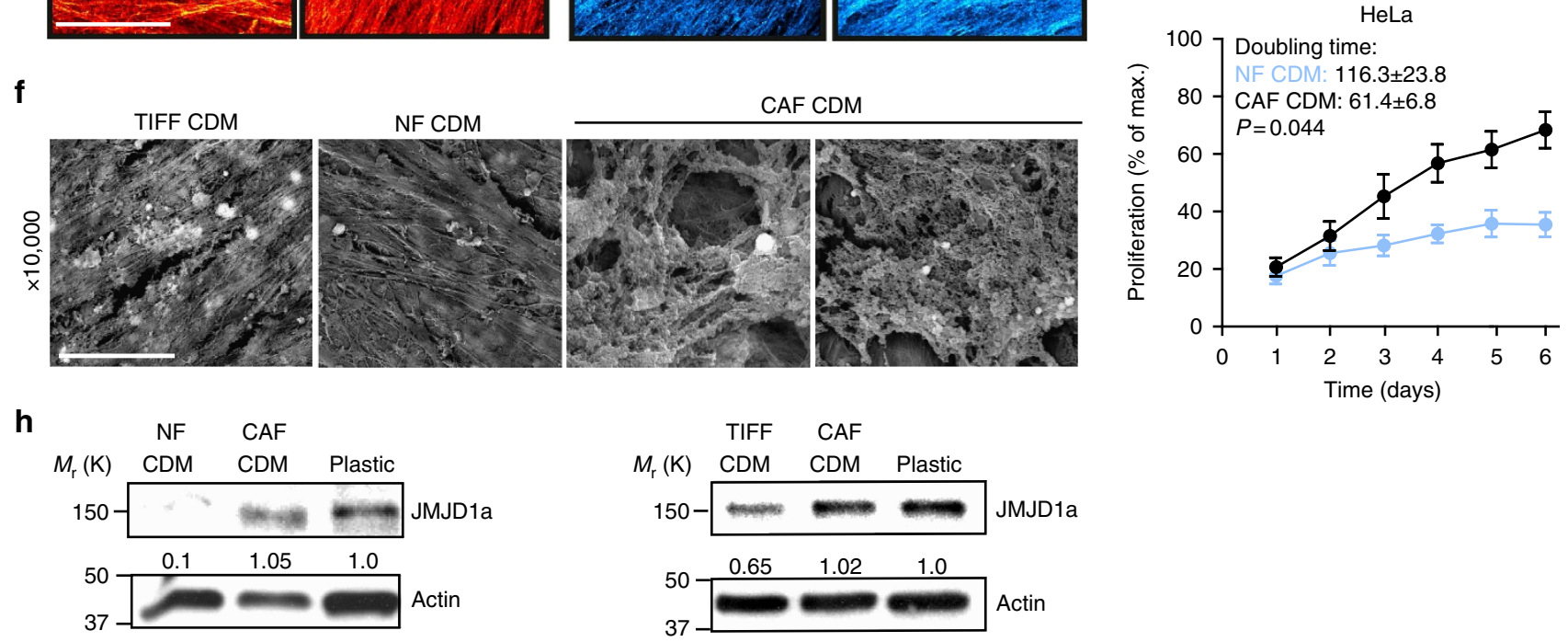

Figure 3 | Patient-derived CAF and NF CDMs are architecturally and functionally distinct. (a,b) Representative western blots showing SMA- $\alpha$ (a) and YAP/TAZ expression (b) in NFs and CAFs isolated from three SCC patients. (c,d) Quantification of YAP (c) and TAZ (d) expression in NFs and CAFs normalized to loading control. Data are mean \pm s.d. (e) Collagen I (red) and fibronectin (blue) staining of patient \#1 and \#3 NF and CAF CDM. Scale bar, $20 \mu \mathrm{m}$. (f) Representative SEM images of TIFF, Patient \#1 NF and CAF CDM. Scale bar, $5 \mu \mathrm{m}$. (g) Proliferation of MDA-MB-231 and HeLa cells on Patient \#1 $\mathrm{NF}$ and CAF CDM. $n(\mathrm{MDA}-\mathrm{MB}-231)=10-11$ and $n(\mathrm{HeLa})=19$ from three independent experiments. Data are mean \pm s.e.m. and $P$ values are calculated from the doubling times. (h) JMJD1a expression in MDA-MB-231 cells cultured on TIFF, NF and CAF CDMs and on plastic. Quantification shows relative JMJD1a amount normalized to loading control. (c,d) Non-parametric Mann-Whitney test was used for statistical analyses in $\mathbf{c}, \mathbf{d}$ and non-paired $t$-test in $\mathbf{g}$.

MDA-MB-231 cells (Supplementary Fig. 4c), suggesting that it might be shuttling between the two compartments. YAP/TAZ transcription factors are well-established mechanosensitive regulators of cell proliferation, such that stiff matrix and cell spreading support YAP/TAZ protein stability and nuclear localization $^{36}$. To study the potential mechanosensitivity of JMJD1a, we investigated JMJD1a localization and levels under conditions known to regulate YAP/TAZ ${ }^{36}$. YAP/TAZ protein 


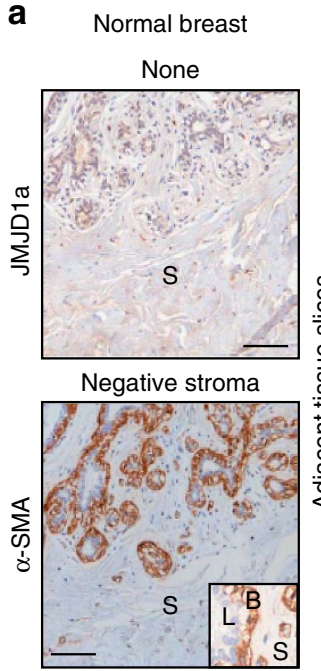

Breast carcinoma
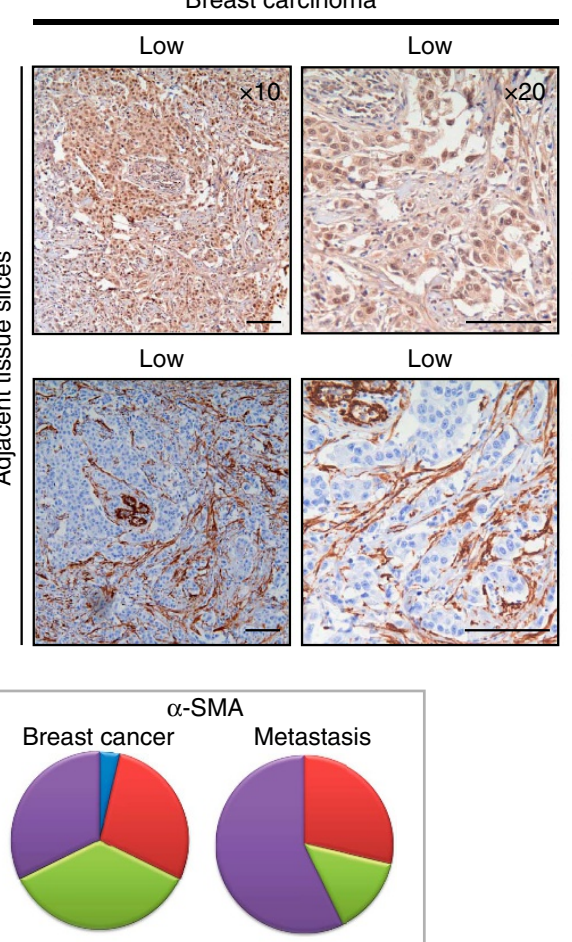

JMJD1a

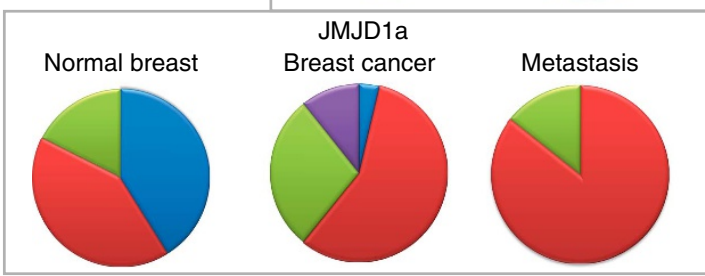

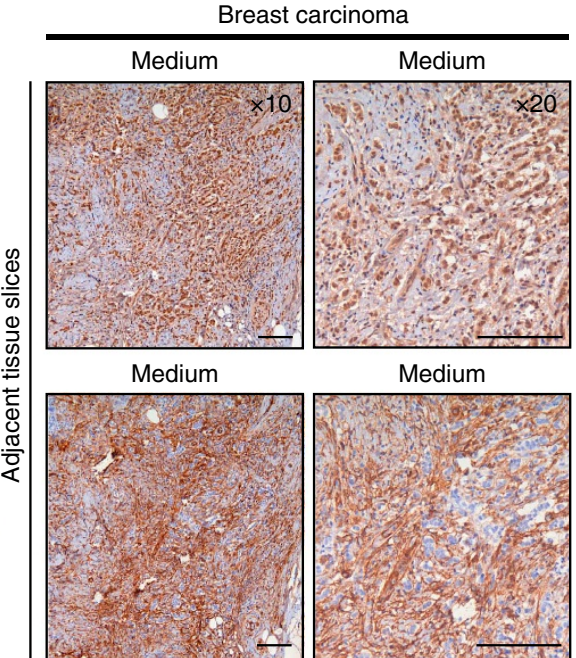

b

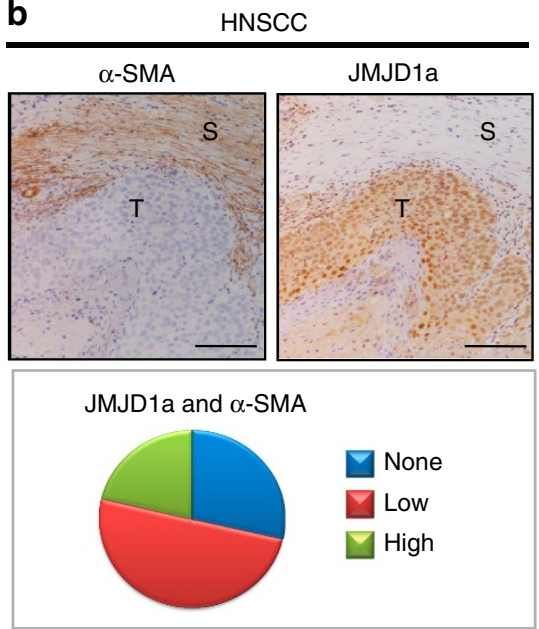

C

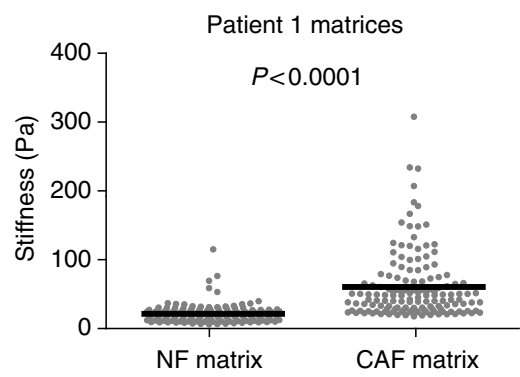

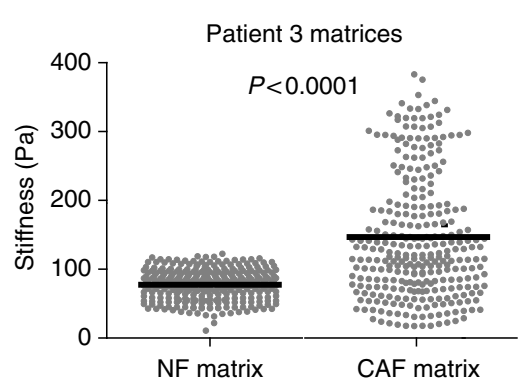

d

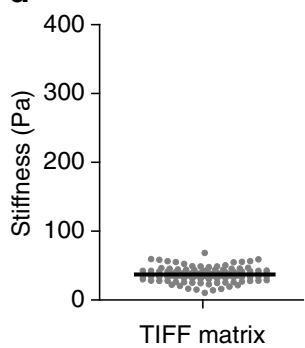

Figure 4 | JMJD1a levels correlate with $\boldsymbol{\alpha}$-SMA-positive stroma in human tumours. (a,b) JMJD1a and $\alpha$-SMA staining from sections of the same normal breast tissue and primary breast carcinomas (a) or HNSCC (b) tissue. For breast carcinomas, examples of the immunostaining of different expression levels and two different magnifications of the same tissue are shown. Scale bar, $200 \mu \mathrm{m}$. S, stroma; T, tumour. Inset in a highlights the $\alpha$-SMA localization in basal cells in the normal mammary gland ( $B$, basal; $L$, luminal). Quantification of the incidence of JMJD1a or $\alpha$-SMA positivity in the analysed samples is shown. (c,d) Stiffness of the patient-derived NF or CAF (c) and TIFF (d) CDMs is expressed by the Young's modulus, which was measured using AFM indentation. Each grey dot represents individual measurement and black line indicates the mean. Non-paired $t$-test was used for statistical analysis. Non-paired $t$-test was used for statistical analysis.

levels are known to be downregulated on soft supports. Interestingly, we found that also JMJD1a stability and therefore the protein levels were reduced when cells were cultured on soft hydrogels (Fig. 5a,b and Supplementary Fig. 4d).

In addition to matrix stiffness, RhoA-signalling, actomyosin contractility and cell spreading are known regulators of YAP/TAZ localization and protein stability ${ }^{16}$. To test whether these ques regulate JMJD1a as well, we plated MDA-MB-231 cells on micropatterns with equal total adhesive surface distributed over a variable spreading area. In cells spreading on $800-\mu \mathrm{m}^{2}$ fibronectin-coated micropatterns (large), both JMJD1a and YAP/TAZ were predominantly nuclear compared with cells spreading on $400-\mu \mathrm{m}^{2}$ (small) fibronectin-coated micropatterns, where both proteins were predominantly cytoplasmic (Fig. 5c,d). Accordingly, we observed that both JMJD1a and YAP/TAZ became cytosolic on lower stiffness hydrogels $(0.5 \mathrm{kPa})$ as soon as 
the cells had fully adhered $(3 \mathrm{~h})$ and were increasingly nuclear on stiffer supports both in MDA-MB-231 and patient SCC cells (Fig. 5e-g and Supplementary Fig. 5a-d), whereas another nuclear protein Son was not mechanosensitive on hydrogels (Supplementary Fig. 5e). Importantly, both JMJD1a and YAP/TAZ were predominantly cytoplasmic in MDA-MB-231 cells grown on soft, normal CDM compared with their nuclear localization on plastic or collagen- and fibronectin-coated plastic (Fig. 5h,i and Supplementary Fig. 5f), and very similar regulation was observed also in the patient-derived SCC cells (Supplementary Fig. 5g). Correspondingly, JMJD1a was prominently cytoplasmic on NF CDM compared with its nuclear localization on CAF CDMs (Fig. 5j), indicating that CDM-induced regulation of JMJD1 localization and levels is similar to the previously established regulation of YAP/TAZ (Supplementary Fig. 5h).

However, unlike YAP/TAZ, JMJD1a localization was not dependent on an intact actin cytoskeleton or Rho-signalling as a

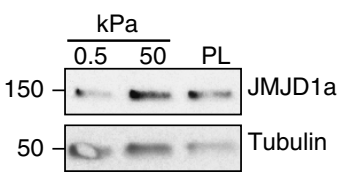

b

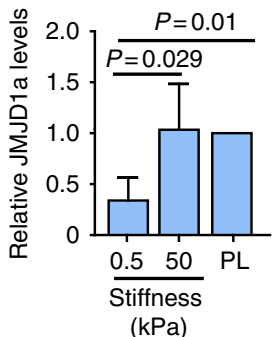

C

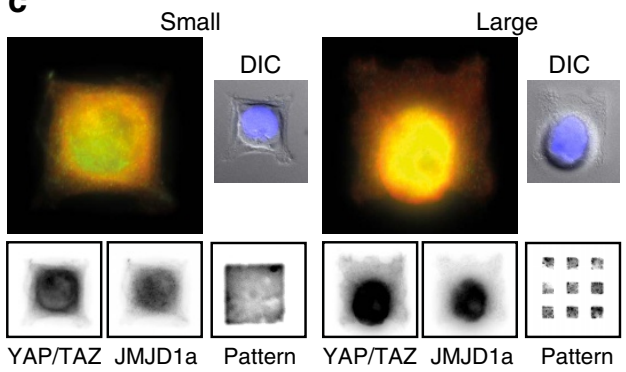

d
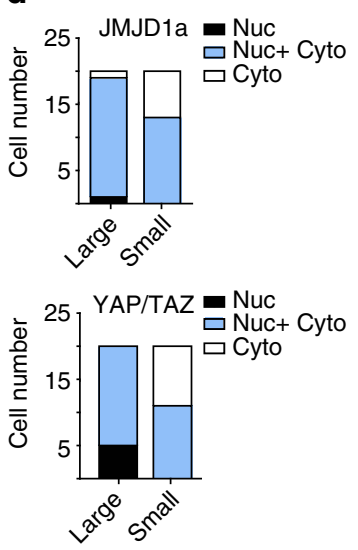

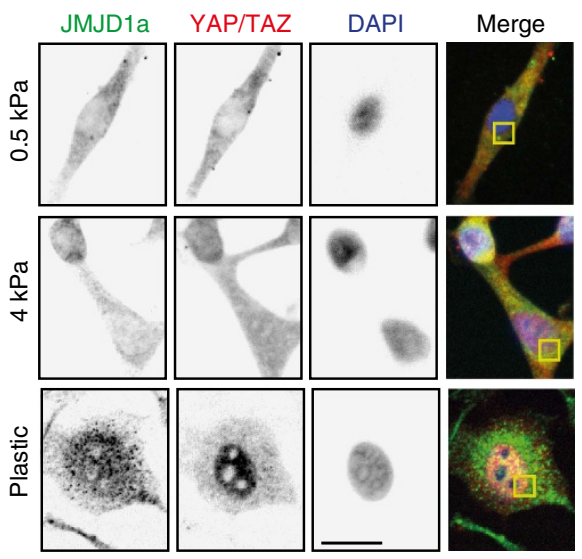

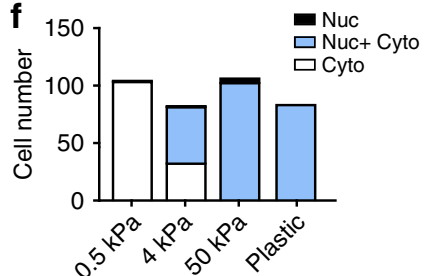

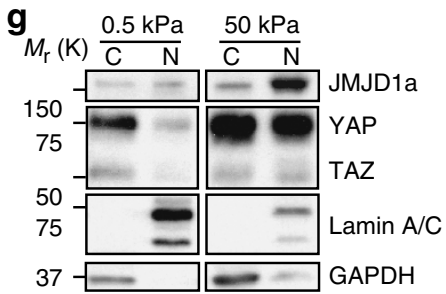

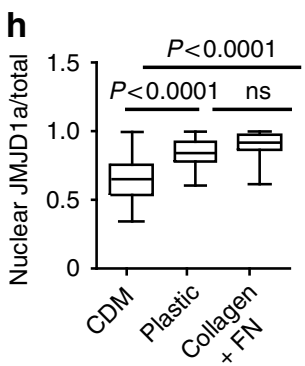
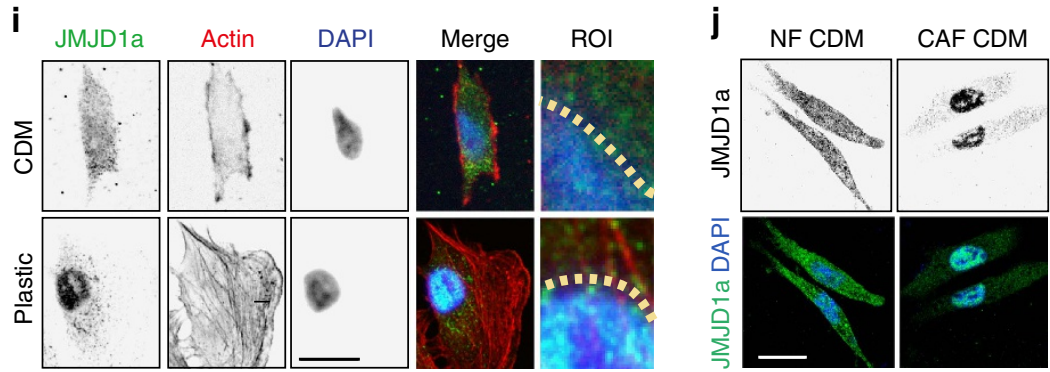

$\mathbf{k}$

CA-Src expression on $0.5 \mathrm{kPa}$

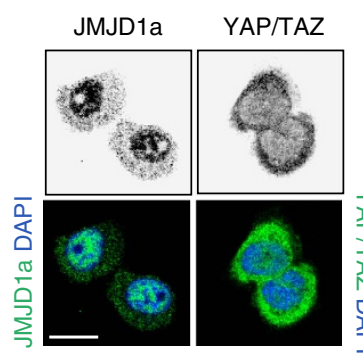

CA-Src expression on plastic

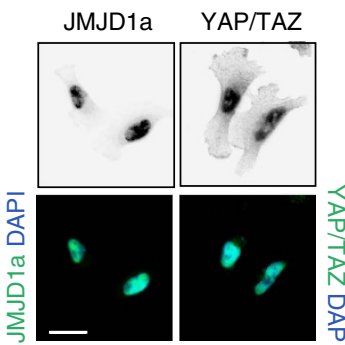

I

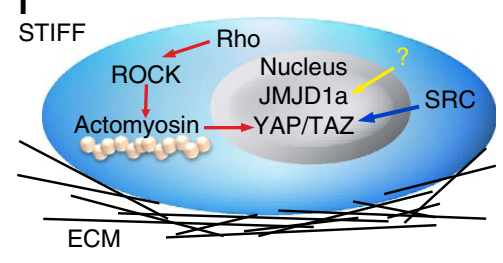

SOFT

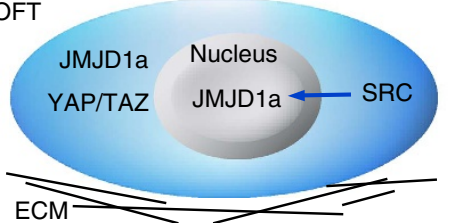


Blebbistatin, Cytochalasin D or ROCK inhibitor Y27632 treatment did not change JMJD1a localization, while YAP/TAZ became predominantly cytoplasmic with all these treatments (Supplementary Fig. 6a). Integrin signalling is known to be important for mechanosignalling ${ }^{37-40}$, albeit the specific requirement for integrin-ECM interaction in YAP/TAZ regulation remains controversial ${ }^{41-43}$. In order to investigate whether integrin $\beta 1$ activity could regulate JMJD1a, we plated cells on integrin $\beta 1$ inactivating (4B4) or activating monoclonal antibodies (12G10) and analysed JMJD1a localization in serumfree conditions. We found that locking integrins into active or inactive conformation was not sufficient to alter JMJD1a localization on soft or stiff (Supplementary Fig. 6b). Furthermore, silencing of integrin $\beta 1$ from MDA-MB-231 cells had no effect on the nuclear localization of JMJD1a, suggesting that JMJD1a nuclear localization is unlikely to be dependent on a specific integrin heterodimer (Supplementary Fig. 6c). Next, we tested whether known integrin downstream effectors, Focal adhesion kinase or Rous sarcoma oncogene cellular homolog (SRC)-kinase, could regulate JMJD1a localization. We found that pharmacological inhibition of Focal adhesion kinase and SRC alone or in combination did not alter JMJD1a localization on plastic but SRC inhibition induced YAP/TAZ translocation to the cytoplasm (Supplementary Fig. 6d). However, expression of constitutively active SRC (CA-SRC) increased tyrosine phosphorylation of GFPJMJDla (Supplementary Fig. 6e) and was sufficient to induce nuclear JMJD1a on soft $0.5 \mathrm{kPa}$ hydrogels (Fig. 5k), indicating that SRC activation is sufficient to support JMJDla nuclear localization on soft. Interestingly, forced expression of CA-SRC did not alter YAP/TAZ localization on soft (Fig. 5k), demonstrating that stiffness-mediated regulatory pathways of YAP/TAZ and JMJD1a localization are distinct (Fig. 5i).

JMJD1a regulates YAP/TAZ expression. JMJD1a and YAP/TAZ levels were significantly reduced on TIFF CDM (Fig. 6a) in line with their cytoplasmic translocation (Supplementary Fig. 5f), and this correlated with reduced transcription of the well-known YAP/TAZ target genes Connective tissue growth factor (CTGF) and Thrombospondin 1 (THBS1) on TIFF CDM compared with plastic (Fig. 6b). Furthermore, we observed that levels of YAP/TAZ and JMJD1a in individual MDA-MB-231 cells correlated significantly (Fig. 6c). Thus, we were interested to investigate the potential link between JMJD1a and YAP/TAZ. Chromatin immunoprecipitation (ChIP) assays revealed that JMJD1a is recruited to TAZ promoter (Fig. 6d, Supplementary Fig. 7a) and, in line with the demethylase activity of JMJD1a, transient JMJD1a silencing increased $\mathrm{H} 3 \mathrm{~K} 9 \mathrm{me} 2$ methylation on the TAZ promoter (Fig. 6e and Supplementary Fig. 7a).
Furthermore, JMJD1a silencing with two independent short interfering RNAs (siRNAs) reduced YAP/TAZ protein and mRNA levels in MDA-MB-231 and patient-derived SCC cells (Fig. 6f-h and Supplementary Fig. 7b-e) as well as expression of YAP/TAZ target genes in MDA-MB-231 (Fig. 6i and Supplementary Fig. 7a). Conversely to JMJD1a silencing, overexpression of JMJD1a-GFP (which localizes to the nucleus similarly to endogenous JMJD1a on plastic, Supplementary Fig. 7f) increased YAP/TAZ levels (Fig. 6j,k) as well as THBS1 and CTGF gene expression (Fig. 61). Importantly, overexpression of JMJD1a was sufficient to increase YAP/TAZ levels even on TIFF-derived CDMs (Fig. $6 \mathrm{~m}$ ) and $4 \mathrm{kPa}$ hydrogels (Fig. 6n), where YAP/TAZ protein-level stability is compromised because of increased cytoplasmic localization and degradation ${ }^{36}$. However, overexpression of wild-type or active YAP mutant (YAP-5SA (ref. 44)) alone was not sufficient to rescue proliferation on MDA-MB-231 cells grown on TIFF-derived CDMs or in JMJD1a-silenced cells, suggesting that additional JMJD1a target genes contribute to the CDM-induced growth inhibition (Supplementary Fig. 7g,h).We also found that silencing of JMJD1a had no effect on either YAP/TAZ nuclear localization (Supplementary Fig. 8a) or phosphorylation of LATS1/2 (Supplementary Fig. 8b), which is a negative regulator of YAP/TAZ protein stability. Furthermore, we could not detect increased YAP (S-127) phosphorylation, which is associated with reduced stability of YAP, upon JMJD1a silencing (Supplementary Fig. 8c) and protein stability of YAP/TAZ was not reduced upon JMJD1a silencing, suggesting that JMJD1a regulates YAP/TAZ on the transcriptional level (Supplementary Fig. 8d). This further demonstrates that JMJD1a is a previously undescribed transcriptional activator of YAP/TAZ expression, and that forced expression of JMJD1a can support YAP/TAZ levels even on soft substrates because of its ability to increase the transcription of YAP/TAZ.

JMJD1a and YAP/TAZ expression correlates in human cancer. We found that JMJD1a and YAP/TAZ expression correlated also in human carcinomas. In a large cohort of primary breast tumours ${ }^{45}$, JMJD1a and YAP/TAZ (the YAP antibody recognizes both transcription factors) were strongly associated with several commonly assessed clinicopathological prognostic factors (Supplementary Tables 1-4 and Supplementary Note 1). In all, $689(94.3 \%)$ and $262(35.8 \%)$ out of the 731 tumours available for JMJD1a staining had positive cytoplasmic and nuclear staining, respectively, and $645(86.5 \%)$ and $514(68.9 \%)$ out of the 746 cancers available for YAP/TAZ staining had positive cytoplasmic and nuclear YAP/TAZ expression.

Fully in line with their correlated expressions in vitro, we found that JMJD1a and YAP/TAZ levels significantly correlated in

\footnotetext{
Figure 5 | Mechanosensitive regulation of JMJD1a on soft and stiff ECM and CDM. (a,b) Representative western blot (a) and quantification (b) showing JMJD1a expression in cells plated on 0.5 and $50 \mathrm{kPa}$ hydrogels and on plastic (PL). Tubulin was used as loading control, $n=4$ (mean \pm s.d.). (c) JMJD1a (red) and YAP/TAZ (green) and DAPI (blue) staining in MDA-MB-231 cells on large $\left(800 \mu \mathrm{m}^{2}\right)$ and small $\left(400 \mu \mathrm{m}^{2}\right)$ spreading area micropatterns (adhesive area is the same). Cell morphology is shown as DIC. (d) Quantification of cytoplasmic and nuclear JMJD1a and YAP/TAZ localization on small and large micropatterns. $n$ (cells) $=20$ per pattern size. (e,f) Immunofluorescence staining showing (e) and quantifying (f) YAP/TAZ and JMJD1a localization on collagen I and fibronectin-coated hydrogels of varying stiffness $(0.5,4$ and $50 \mathrm{kPa}$ ) and on plastic. Scale bar, $10 \mu \mathrm{m}$. (g) Representative western blot showing JMJD1a and YAP/TAZ nuclear (N) and cytoplasmic (CP) localization in cells plated on 0.5 and $50 \mathrm{kPa}$ hydrogels. Lamin A/C and GAPDH were used as fractionation controls. (h,i) Immunofluorescence staining quantification (i) and representative images (h) of JMJD1a localization in cells plated on TIFF CDM, plastic or collagen and fibronectin ligands $\left(2.5 \mu \mathrm{g} \mathrm{ml} \mathrm{l}^{-1}\right.$ collagen and $2.5 \mu \mathrm{g} \mathrm{ml} \mathrm{I}^{-1}$ fibronectin). Nuclear localization of JMJD1a was quantified with the CellProfiler software. $n$ (cells): $C D M=74$ cells; plastic $=33$ cells and collagen $+F N=61$. Scale bar, $10 \mu \mathrm{m}$. (j) Representative immunofluorescence images showing JMJD1a localization in MDA-MB-231 cells growing on NF and CAF CDMs for 3 days. Scale bar, $10 \mu \mathrm{m}$. (k) YAP/TAZ and JMJD1a localization in CA-SRC-expressing MDA-MB-231 cells growing on soft $0.5 \mathrm{kPa}$ hydrogels or on plastic. Representative images from three independent experiments. Scale bar, $10 \mu \mathrm{m}$. (I) Model of distinct mechanotransductional regulation of YAP/TAZ and JMJD1a on soft and stiff. Red arrows indicate the pathway, which we and others have shown to regulate YAP/TAZ nuclear localization. Blue arrow indicates the SRC kinase-mediated and stiffness-dependent regulation of JMJD1a and YAP/TAZ.
} 
clinical tumour samples available for both stainings (Fig. 7a). Furthermore, nuclear localization of YAP/TAZ was significantly associated with nuclear JMJD1a in tumours (Fig. 7a). A similar correlation was found also on the mRNA level. Integrated data from multiple gene-expression-profiling studies of breast ductal carcinoma (720 patients) and oral and skin SCC (47 patients) showed a significant positive correlation between JMJD1a $(K D M 3 A)$ and TAZ (WWTR1) gene expression (Supplementary
Fig. 9). Taken together, these data reveal a previously unknown mechanosensitive relationship between JMJD1a and YAP/TAZ expression both in vitro in cancer cell lines and in a large number of clinical patient samples from the same cancer types.

\section{Discussion}

We describe a new mechanism of ECM-mediated control of cancer cell proliferation. Our results indicate that epigenetic
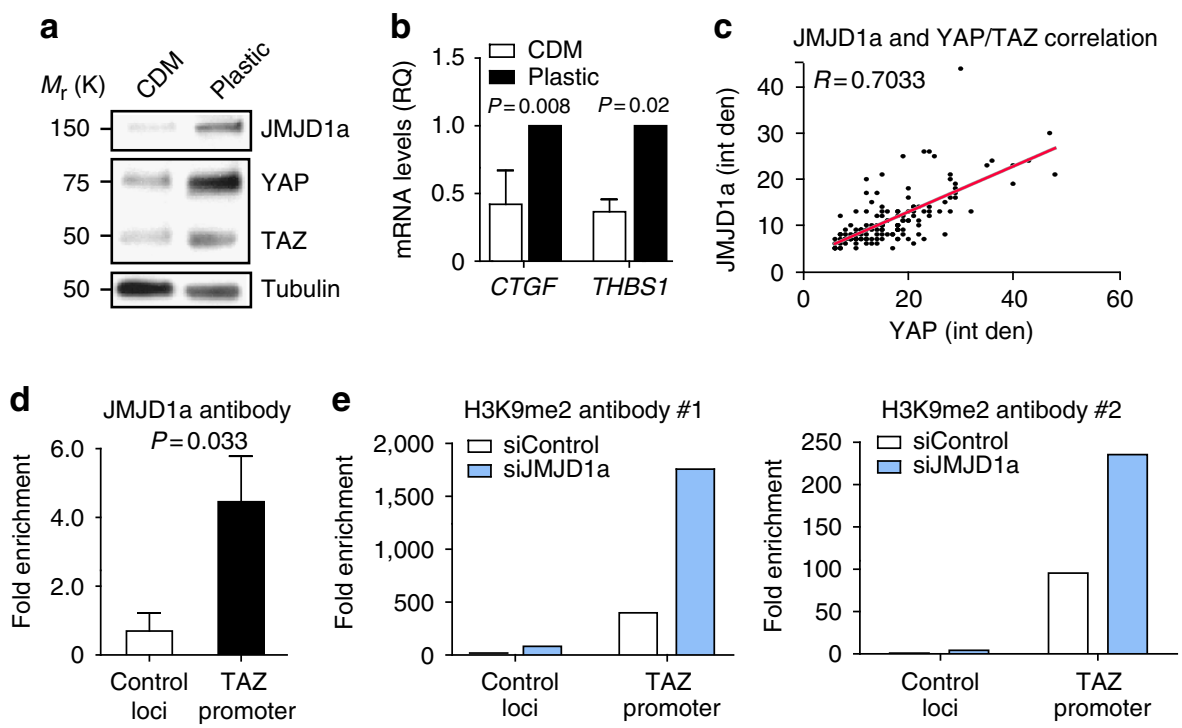

f
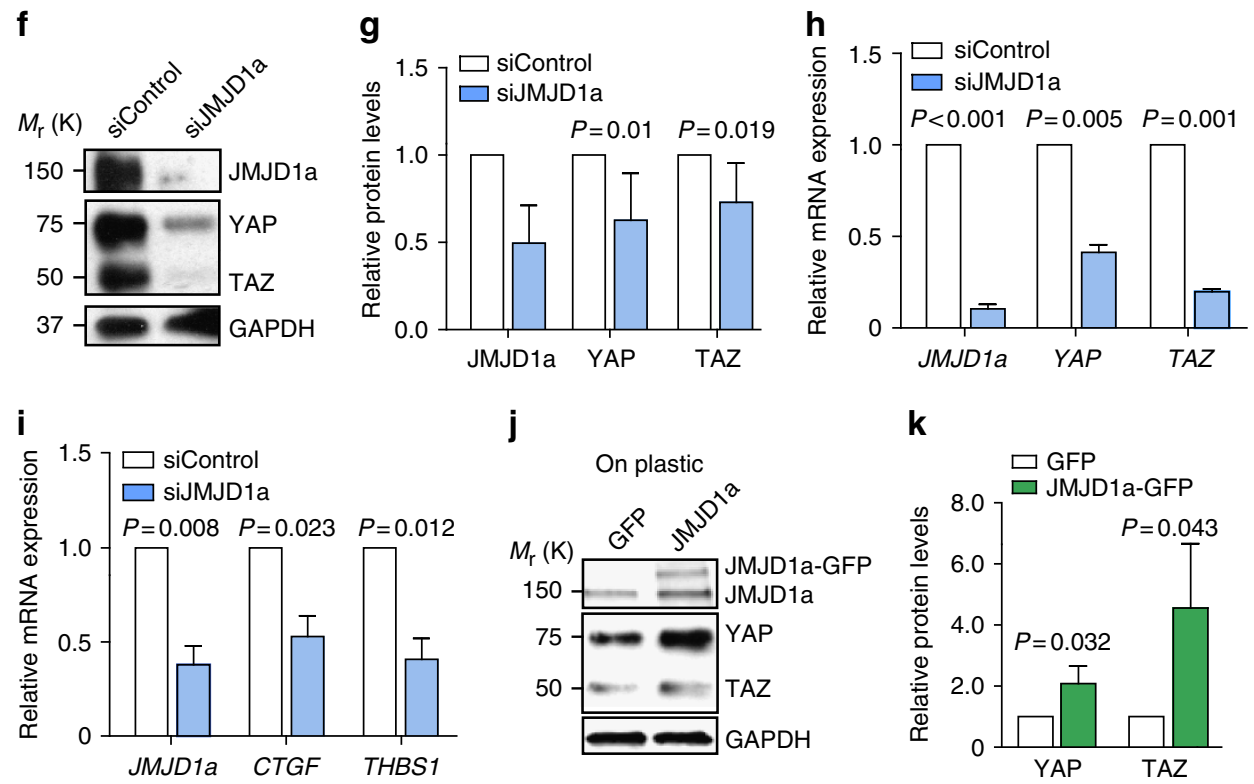

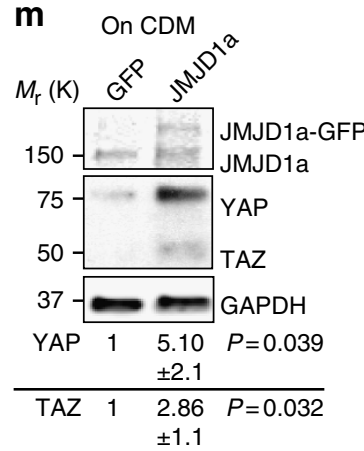

$\mathbf{n}$

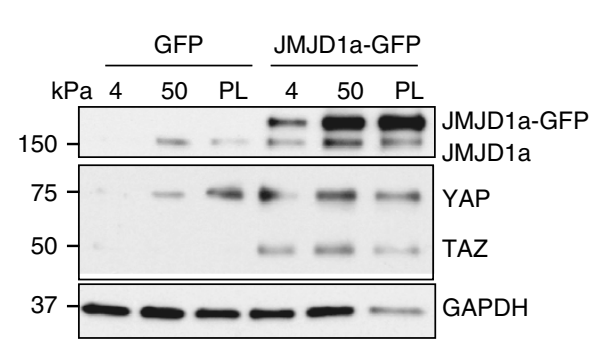


modifier JMJD1a is a mechanosensitive regulator of the transcription of many genes, including YAP and TAZ (WWTR1) and of cancer cell proliferation (Fig. $7 \mathrm{~b}$ ). In cancer, changes in ECM composition and mechanical properties of the tumour microenvironment are the focus of intense research, as matrix stiffening and appearance of CAFs in the tumour stroma correlate with cancer progression and metastasis ${ }^{22,46}$. However, the mechanisms of how normal tissue could function to suppress or restrict tumour growth are much less well understood. We propose that ECM generated by NFs is growth-restrictive to cancer cells in vitro and has the capacity to inhibit the growth of malignant carcinomas in vivo. Mechanistically, the soft normal ECM triggers the translocation of histone demethylase JMJD1a from the nucleus to the cytoplasm. This is followed by JMJD1a downregulation on the protein and mRNA levels as well as a downregulation of proliferation-inducing genes, including YAP/TAZ (WWTR1). Thus, our results define an unprecedented level of regulation of cancer cell proliferation, where the mechanosensitive control of JMJD1a regulates its nuclear availability to regulate transcription of proliferative genes.

By comparing CDMs generated by NFs and CAFs from the same individual, we found that normal CDM, but not CAF $\mathrm{CDM}$, had a strong growth-inhibitory influence on primary patient-derived HNSCC cells. CAF-derived CDM exhibited altered architecture as well as increased stiffness compared with the NFderived CDM, in line with what has been demonstrated for carcinomas in patients ${ }^{34,47}$. Thus, it appears that normal stroma possesses tumour-suppressive functions that are lost in the reactive cancer-associated stroma. We found that tumour-suppressive capacity of the normal stroma is connected to stiffness but is likely to be mediated by changes also in matrix architecture and composition. This observation could be linked to the important but largely unexplored fact that, even though cancer incidences are increasing globally, most people never get cancer. Pathologists frequently observe numerous microscopically detectable cancerous foci in the mammary gland or prostate of people who never develop advanced clinical cancer ${ }^{4}$. Furthermore, several studies (reviewed in ref. 48) have demonstrated that normal tissue has the capacity to restrict the growth of cancer cells and to determine the cancer incidence of transplanted normal tissue.

JMJD1a is regulated by hypoxia ${ }^{28,49}$; however, its regulation under normoxic conditions has not been investigated. We find that matrix stiffness regulates the subcellular localization of JMJD1a and that on soft matrices JMJD1a is cytoplasmic and becomes downregulated. JMJD1a has been found to be upregulated in several cancer types ${ }^{50,51}$ and to be important for cancer cell proliferation ${ }^{52}$. In human bladder carcinomas, JMJD1a overexpression could already be detected at early-stage carcinomas before the generation of hypoxic conditions ${ }^{27}$. These data suggest that JMJD1a contributes to cancer progression even under normoxia and that JMJD1a levels are regulated early in cancer progression. We find in clinical samples that a vast majority of human breast carcinomas express elevated levels of JMJD1a compared with normal tissue. Furthermore, the stroma in these tumours is positive for the CAF marker $\alpha$-SMA. Thus, JMJD1a expression also correlates with stromal changes in vivo.

Breast cancer expression of JMJD1a and YAP/TAZ were strongly associated with several unfavourable prognostic factors in the breast cancer cohort ${ }^{45}$, suggesting clinical relevance. Tumour JMJD1a and YAP/TAZ expression did not, however, influence survival, and their expression was not associated with an increased frequency of axillary nodal metastases. Therefore, while JMJD1a and YAP/TAZ favour cancer proliferation, their expression might not contribute to metastasis.

Regulation of the YAP/TAZ pathway has been intensely investigated in development and cancer ${ }^{53}$. However, the focus has been predominantly on how YAP/TAZ nuclear localization and protein stability are controlled and how YAP and TAZ regulate the transcription of their target genes together with DNA-binding proteins of the TEAD (TEA/ATTS domain) family ${ }^{54}$. We find that JMJD1a regulates YAP/TAZ transcription in a stiffness-sensitive manner, and that forced expression of JMJD1a is sufficient to support YAP/TAZ levels also on soft substrates. This is to the best of our knowledge the first mechanistic demonstration for mechanosensitive regulation of an epigenetic regulator enzyme as well as of YAP/TAZ on the transcriptional level.

\section{Methods}

Cell lines and cell culture. SCC cell lines and CAFs were isolated from different parts of head and neck region (Supplementary Fig. 1a). The UT-SCC-54A (Patient \#2) cell line was established from a primary T2N0M0 Grade 1 tumour of the buccal mucosa Grade 1. The donor (non-smoker) did not excessively consume alcohol. The UT-SCC cell line was established using the explant method from primary, recurrent or metastatic tumours. The fibroblasts were removed by selective trypsinization as previously described in ref. 55 . The CAF population was identified by SMA- $\alpha$ expression by western blotting. NFs were isolated from a normal, non-cancerous region. MDA-MB-231 human breast adenocarcinoma cells (American Type Culture Collection, ATCC), primary fibroblasts and SCC (UT-SCC-54A) cells were maintained in DMEM (4500 $\mathrm{mgl}^{-1}$ glucose, Sigma) containing $1 \%$ non-essential amino acids (Sigma), 1\% L-Glutamine (Gibco) and $10 \%$ fetal bovine serum (FBS). HeLa cells (ATCC) were maintained in DMEM (1000 $\mathrm{mgl}^{-1}$ glucose) supplemented with $10 \%$ FBS and $1 \%$ L-glutamine. The usage of human tissue to derive cell lines was approved by the Finnish national authority for medicolegal affairs (Dnro 889/04/047/08) and regional ethics committee of University of Turku (Dnro 146/2007).

Cell-derived matrices. CDMs were prepared as described in ref. 20. Coverslips were coated with $0.2 \%$ gelatin for $60 \mathrm{~min}$ at $37^{\circ} \mathrm{C}$ (Sigma G1393 in PBS), followed by crosslinking with $1 \%$ glutaraldehyde for $30 \mathrm{~min}$ at room temperature (RT). Crosslinker was quenched with $1 \mathrm{M}$ glycine for $20 \mathrm{~min}$ at RT. Gelatin-coated coverslips were incubated with medium before seeding 50,000 cells on coverslips. Ascorbic acid treatment $\left(50 \mu \mathrm{g} \mathrm{ml}^{-1}\right)$ was started when the cell layer was confluent.

Figure 6 | JMJD1a regulates YAP/TAZ transcription (a) Representative western blot showing YAP/TAZ expression in MDA-MB-231 on CDM and on plastic. (b) Taqman qRT-PCR of CTGF $(n=4)$ and THBS1 $(n=5)$ mRNA levels in MDA-MB-231 cells grown on CDM or on plastic. (c) Quantification of JMJD1a and YAP/TAZ staining intensity from immunofluorescence images of MDA-MB-231 cells on plastic. Intensity (int den) was quantified using the CellProfiler software. $n$ (cells) $=146$. $R$-value indicates correlation. (d) ChIP showing the binding of JMJD1a to the TAZ promoter. Analysis was performed by SYBR green-based detection and fold increase in signal relative to the background signal (IgG control antibody) is shown. $n=3$ (mean \pm s.d.). Paired $t$-test was used to calculate $P$ value. (e) ChIP showing H3K9me2 levels on TAZ promoter of siControl and siJMJD1a_3-transfected MDA-MB-231 cells. ChIP was performed with two independent $\mathrm{H} 3 \mathrm{~K} 9 \mathrm{me} 2$ antibodies. Analysis was performed by SYBR green-based detection and fold increase in signal relative to the background signal (IgG control antibody) is shown. Representative results from two independent experiments. (f,g) A representative western blot (e) and quantification (f) showing YAP/TAZ expression in JMJD1a-silenced MDA-MB-231 cells after 3 days of silencing, $n=7$. (h) Taqman qRT-PCR of JMJD1a (KDM3A), CTGF and THBS1 mRNA levels in JMJD1a-silenced MDA-MB-231 cells, $n=4$. (i) Taqman qRT-PCR of YAP and TAZ mRNA levels in JMJD1a siRNA-transfected MDA-MB-231 cells. $n(J M J D 1 a$ and TAZ) $=4, n(Y A P)=3$. (j,k) Representative western blot (i) and quantification (j) showing YAP/TAZ expression in JMJD1a-overexpressing MDA-MB-231 cells normalized to loading control, $n=4$. (I) Taqman qRT-PCR of JMJD1a (KDM3A), CTGF and THBS1 mRNA levels in JMJD1a-overexpressing MDA-MB-231 cells, $n=4$. ( $\mathbf{m})$ A representative western blot and quantification of YAP/TAZ expression on TIFFderived CDM in GFP control and JMJD1a-GFP-overexpressing cells after 4 days on CDM. (n) Representative western blot of JMJD1a and YAP/TAZ expression in GFP control and JMJD1a-overexpressing MDA-MB-231 cells plated on 4 or $50 \mathrm{kPa}$ hydrogels or on PL. 


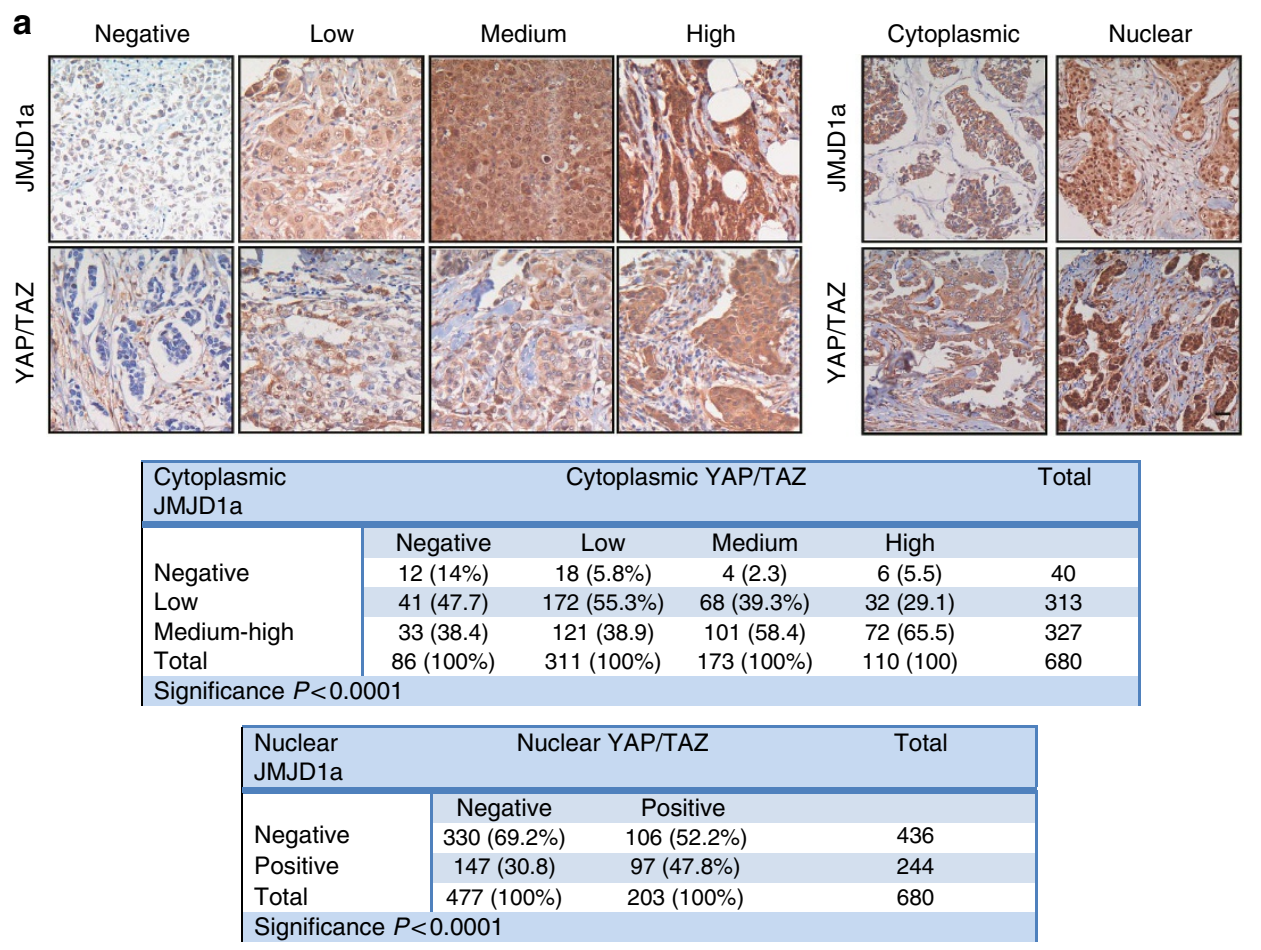

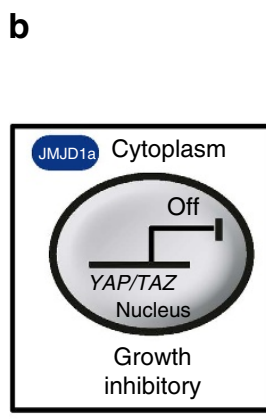

- Loose and soft ECM
- Low/cytoplasmic JMJD1a

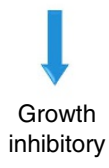

Normal stroma

ECM derived from normal fibroblasts
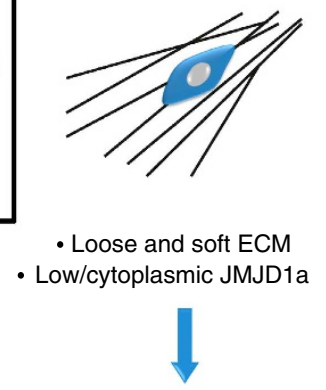

inhibitory

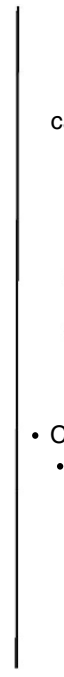

Reactive stroma

ECM derived from cancer-associated fibroblasts
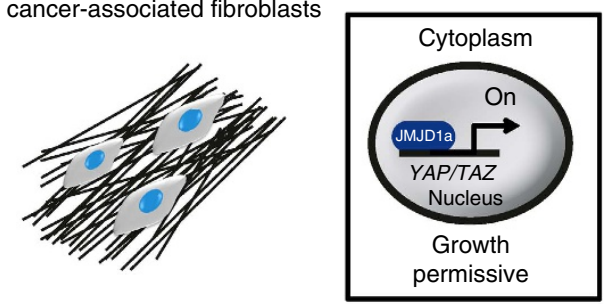

Compacted and stiff ECM

- High/nuclear JMJD1a

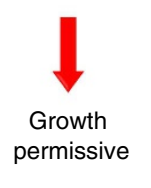

Figure 7 | JMJD1a and YAP/TAZ expression correlates in human carcinomas. (a) Example images for the scoring of immunohistochemical stainings for JMJD1a and YAP/TAZ expression from primary breast cancer tissue samples. Scale bar, $100 \mu \mathrm{m}$. The tables show the association between cytoplasmic and nuclear expression of JMJD1a and YAP/TAZ. The $\chi^{2}$-test was used for the statistical analyses. (b) A proposed model for the normal and cancer-associated matrix-induced control of cancer cell proliferation via mechanosensitive regulation of JMJD1a and transcriptional regulation of YAP/TAZ.

Ascorbic acid-containing medium was changed every day for 10 (TIFFs) — or 21 days (primary fibroblast cell lines). After ascorbic acid induction, cells are removed by extraction buffer treatment ( $20 \mathrm{mM} \mathrm{NH}_{4} \mathrm{OH}, 0.5 \%$ Triton-X in PBS). Remaining DNA was removed by $10 \mu \mathrm{M}$ DNAse treatment for $1 \mathrm{~h}$ at $37^{\circ} \mathrm{C}$.

Proliferation assays. Proliferation assays for the MDA-MB-231 and HeLa cells were performed using Incucyte-FLR or Incucyte-ZOOM live-cell microscopy incubator (Essen Bioscience). In all, 5,000 of the cancer cells to be analysed (GFP-positive HeLa or MDA-MB-231 cells) were plated on 24 wells (on CDM or on plastic) and imaged hourly over several days. Stably GFP-positive MDA-MB-231 and HeLa cells were used throughout to facilitate determining the cell number at the indicated time points on CDM. The doubling times were calculated using GraphPad (nonlinear regression curve - exponential growth equation; $Y=Y 0^{\star} \exp \left(k^{\star} X\right)$ ]. Since the SCC cells were not GFP-positive, their proliferation was analysed by counting the nuclei (4,6-diamidino-2-phenylindole (DAPI) staining). Cells were plated on CDMs or on plastic in identical numbers. After 4 days, the cells were fixed and stained with DAPI. Images were taken with a $\times 4$ objective. Four images were taken from each well and each condition had three to four wells. The amount of nuclei/image was quantified with the CellProfiler software.

Illumina microarray. The Illumina gene expression data were normalized using the quantile normalization method in Bioconductor (http://www.bioconductor.org) and log-transformed (base 2). Log ratios of the intensities were calculated between the paired samples: ECM versus plastic ( $n=2$ pairs) and ECM to plastic versus plastic ( $n=3$ pairs) in both MDA-MB-231 and HeLa samples. Systematic differences in gene expression between the CDM and CDM to plastic conditions were identified using the rank product algorithm (Bioconductor RankProd package) separately for the MDAMB-231 and HeLa cell lines. Genes with false discovery rate below 0.05 and change in the same direction in both cell lines were considered as differentially expressed.

Quantitative RT-PCR. For quantitative reverse transcriptase (qRT-PCR), total RNA was extracted using an RNAeasy Mini Kit (Qiagen) and converted to cDNA using a high-capacity cDNA Reverse Transcription Kit (Applied Biosystems) according to the manufacturer's instructions. TaqMan probe-based quantitative real-time PCR was 
analysed using Real-Time PCR HT7900 (Applied Biosystems). Glyceraldehyde-3phosphate dehydrogenase (GAPDH) was used as endogenous control. The qPCR primers and universal probe library probes used are listed in Supplementary Table 5.

mRNA sequencing. For the mRNA sequencing, NFs and CAFs were collected from 10-cm cell culture dishes, lysed in RLT lysis buffer (Qiagen) and RNA was isolated using the NucleoSpin Kit (Macherey-Nagel). Sequencing was performed with Illumina HiSeq2500 instrument using single-end sequencing chemistry with 50-bp read length.

Chromatin immunoprecipitation. In order to collect the cells, the growth medium was removed and the culture was washed three times with PBS. We collect minimum two million cells per antibody. Protein-chromatin complexes were crosslinked with $1 \%$ paraformaldehyde (PFAH) for $10 \mathrm{~min}$ at RT (in case of H3K9me2). For JMJD1a, ChIP cells were crosslinked with disuccinimidyl glutarate (Sigma) for $45 \mathrm{~min}$ followed by $10 \mathrm{~min}$ incubation in 1\% PFAH. Disuccinimidyl glutarate was used for JMJD1a ChIP since crosslinkers with longer linker arms have been used for JMJD1a ChIP ${ }^{49}$. Crosslinking reaction was stopped with $0.125 \mathrm{M}$ Glycine by 5-min incubation. Cells were detached with scraper, collected by centrifugation $(300 \mathrm{~g}, 10 \mathrm{~min})$ and washed once with pre-lysis buffer I $(10 \mathrm{mM}$ EDTA, $0.5 \mathrm{mM}$ EGTA, $10 \mathrm{mM}$ HEPES and $0.25 \%$ Triton X) and once with pre-lysis buffer II (1 mM EDTA, $0.5 \mathrm{mM}$ EGTA, $10 \mathrm{mM}$ HEPES and $100 \mathrm{mM} \mathrm{NaCl})$. Cells were lysed by suspending the pellet in $1 \mathrm{ml}$ of lysis buffer $(10 \mathrm{mM}$ EDTA, $50 \mathrm{mM}$ Tris- $\mathrm{HCl}$ and $0.5 \%$ SDS, protease inhibitors) and incubating samples for $10 \mathrm{~min}$ on ice. DNA was fragmented by $4 \times 5 \mathrm{~min}$ sonication ( $30 \mathrm{~s}$ on, $30 \mathrm{~s}$ off). Sheared chromatin was spun down at $10,000 \mathrm{~g}$ for $1 \mathrm{~min}$ at $4{ }^{\circ} \mathrm{C}$. Chromatin was aliquoted so that $\sim 100 \mu \mathrm{g}$ of chromatin was used per antibody. Dilution buffer (1\% Triton$\mathrm{X} 100,2 \mathrm{mM}$ EDTA, $150 \mathrm{mM} \mathrm{NaCl}$ and $20 \mathrm{mM}$ Tris- $\mathrm{HCl}$ ) was used to adjust the total volume to $600 \mu \mathrm{s}^{-1}$. Chromatin was pre-cleared by adding $50 \mu \mathrm{l}$ of Dynabeads (10003D, Life Technologies) and incubating for $1 \mathrm{~h}$ at $4{ }^{\circ} \mathrm{C}$ on rotation. Beads were removed and $5 \mu \mathrm{g}$ of JMJD1a (sc-376608, Santa Cruz), H3K9me2 antibody \#1 (ab1220, Abcam) H3K9me2 antibody \#2 (C15410060, Diagenode) or mouse control IgG (sc-2025, Santa Cruz) antibody was added per sample, followed by overnight incubation at $+4^{\circ} \mathrm{C}$ on rotation. In order to collect the immunocomplexes, $50 \mu \mathrm{l}$ of Dynabeads was added per sample followed $2 \mathrm{~h}$ incubation at $+4^{\circ} \mathrm{C}$ on rotation. Washes were performed once with low-salt wash buffer $(2 \mathrm{mM}$ HEPES, $150 \mathrm{mM} \mathrm{NaCl}, 1 \%$ Triton X, $1 \mathrm{mM}$ EDTA and $20 \mathrm{mM}$ Tris$\mathrm{HCl}$ ), four times with high-salt wash buffer (50 mM HEPES, $500 \mathrm{mM} \mathrm{NaCl}, 0.1 \%$ SDS, $1 \%$ Triton X and $1 \mathrm{mM}$ EDTA) and twice with TE buffer $(10 \mathrm{mM}$ Tris- $\mathrm{HCl}$ and $1 \mathrm{mM}$ EDTA $\mathrm{pH}$ 8.0). DNA was collected to elution buffer by 15 -min incubation at RT, and proteins were removed by Proteinase $\mathrm{K}$ incubation at $55^{\circ} \mathrm{C}$ overnight. DNA was isolated by standard phenol-chloroform-isoamyl extraction (25:24:1 phenol-chloroform-isoamyl pH 7.8). Real-time PCR was carried out using $5 \mu \mathrm{g}$ of DNA per reaction and SYBR green-based detection (Qiagen RT2 SYBR Green ROX qPRC Mastermix cat: 330520) according to the manufacturer's instructions. EpiTect ChIP qPCR Primer Assay For Human WWTR1 (TAZ) was used: NM_015472.3 $(+) 01 \mathrm{~Kb}$ : GPH1023327( + )01A for JMJD1a ChIP and NM_015472.3 ( - )04Kb: GPH1023327( - )04A for H3K9me2 ChIP. Negative control primers \#1 and \#2, which recognize untranslated genomic regions, were used (Control\# 1 forward $5^{\prime}$-CTGTACCTGGGGTTCATTCAT- $3^{\prime}$ and reverse 5'-CAGTAAGCCGTTCACTCTCACA-3'; Control $\# 2$ forward 5'-ATCACACTGC AAAAATCCAGAA- $3^{\prime}$ and reverse $5^{\prime}$-TCACTTCTTTAACTGGCCTTGA- $3^{\prime}$ ). Fold enrichment was calculated by subtracting the background signal (Ct (IP) $-\mathrm{CT}(\mathrm{IgG}$ control)) and calculating the fold enrichment (2-DDCt).

Western blotting and phosphotyrosine pull-downs. Standard western blotting techniques and Amersham ECL Plus Western blotting reagent were used. Following antibodies were used: JMJD1a (12835-1-AP, Proteintech, USA, 1:1,000), YAP/TAZ (sc-101199, Santa Cruz Biotechnology, USA, 1:500), GAPDH (5G4, HyB test 1:5,000), Tubulin (12G10, Hybridoma bank, 1:5,000), Lamin A/C (sc-7292, Santa Cruz Biotechnology, 1:1,000), H3K9me2 (\#7658, Cell Signaling, 1:1,000), Histone 3 (\#4499, Cell Signaling, 1:1,000), actin (clone AC-74, Sigma, 1:1,000), alpha-SMA (A2547, Sigma, 1:1,000) and antiphosphotyrosine antibody (APY03, Cytoskeleton, 1:1,000). For the phosphotyrosine pull-downs, MDA-MB-231 cells co-transfected with GFP-JMJD1a and CA-Src or empty vector were lysed and subjected to pulldown with beads of APY03 covalently coupled to sepharose (Anti-Phosphotyrosine Affinity Beads \# APY03-Beads (Cytoskeleton) according to the manufacturer's instructions. The pulldowns and cell lysate were resolved on SDS-PAGE and subjected to western blot analysis with anti-GFP antibody (Abcam \#1218).

Uncropped scans of the most important blots are provided as Supplementary Fig. 10.

Immunofluorescence. Cells were fixed with $4 \% \mathrm{PFAH}$ for $10 \mathrm{~min}$ at RT and simultaneously permeabilized and blocked with $0.3 \%$ Triton in $30 \%$ horse serum (Gibco) for $10 \mathrm{~min}$ at RT. Following antibodies and antibody dilutions were used: JMJD1a (12835-1-AP, Proteintech, 1:100), YAP/TAZ (sc-101199, Santa Cruz Biotechnology, 1:75), JMJDla (sc-376608, Santa Cruz Biotechnology, 1:100), Atto-Phalloidin-647N (65906, Sigma, 1:200), Collagen I (NB600-408, Novus, 1:100) and Fibronectin (F3648, Sigma, 1:400).
Microscopy and image analysis. Immunofluorescence stainings were imaged with Zeiss spinning disc confocal (Orca-ER camera (Hamamatsu Photonics), Plan-Neofluar $\times 40$ or $\times 63$ oil/1.4 numerical aperture (NA) objective (Carl Zeiss)) or with Zeiss LSM780 laser scanning confocal $(\times 63$ water/1.4 NA objective (Carl Zeiss)). Nuclear localization of JMJD1a was quantified with the CellProfiler image analysis software. The nucleus was defined with the DAPI staining. For JMJD1a nuclear localization, total JMJD1a and nuclear JMJD1a were quantified.

Linescan analysis of CDMs. Fibre organization of Collagen 1 and fibronectinstained CDMs were analysed in ImageJ by drawing a line along the image (three lines per image, 10 images in total). Staining intensity was quantified along the line $(\rightarrow$ analyse $\rightarrow$ blot profile). $x$ and $y$ coordinates were exported $(\rightarrow l i s t)$ and exported to GraphPad. Graph displays the average and s.d. of staining intensity along the line.

Atomic force microscopy. NF- and CAF-derived matrices were grown as described above. The stiffness of CDMs was evaluated with JPK AFM with CellHesion module (JPK Instruments) on Zeiss LSM510 microscope (Carl Zeiss Microscopy). Silicon AFM probes with $1 \mathrm{~N} \mathrm{~m}^{-1}$ cantilever and spherical $45-\mu \mathrm{m}$ polystyrene particle tip (Novascan Technologies) were used on force measurements, where Young's module was detected. During the measurements, matrices were kept in PBS at $37^{\circ} \mathrm{C}$ using the BioCell module (JPK). Before the force measurements, AFM cantilevers were calibrated with the instrument's calibration programme by measuring deflection sensitivity and spring constant. Force measurements of matrix samples were performed on three conditions of a matrix using four random locations per matrix and grids of $5 \times 5$ on each location. As control for approach measurements, the region of interest was visualized with a charge-coupled device camera, mounted on the microscope. Probe extension was carried out with the following settings: travelling distance of $50 \mu \mathrm{m}$, speed $5 \mu \mathrm{m} \mathrm{s}^{-1}$ and sampling rate $205 \mathrm{~Hz}$. We obtained force distance curves by indenting with forces up to $30 \mathrm{nN}$ (maximal indentation $<20 \mu \mathrm{m}$ in all experiments). The gel thickness was with $\sim 10-15 \mu \mathrm{m}$ sufficiently thick to assume a Hertz model ${ }^{56}$, and the fits used a Nelder-Mead algorithm to determine the Young's modulus and the touching point. The Hertz model did fit the full data range with a high confidence $\left(R^{2}>0.95\right.$ for $88 \%$ of the measurements).

Scanning electron microscopy. Matrices were fixed with $2 \%$ glutaraldehyde in $0.1 \mathrm{M} \mathrm{NaCac}$ buffer, $\mathrm{pH} 7.4$ for $20 \mathrm{~min}$ and washed twice with NaCac buffer. Samples were sonicated for 30-60 min at RT in $1 \% \mathrm{OsO}_{4}$ in $0.1 \mathrm{M} \mathrm{NaCac}$ and washed twice with $0.1 \mathrm{M} \mathrm{NaCac}$ buffer. Matrices were dehydrated by 3 min incubation once with $50 \%$, once with $70 \%$ and once with $96 \% \mathrm{EtOH}(\mathrm{A})$ and finally twice with absolute EtOH (Aa) for 5 min. Finally, matrices were covered with hexamethyldisilazane. Coverslips were mounted to aluminium specimen stubs using double-sided graphite tape and addition of one drop of graphite glue to the edge of the coverslip.

Hydrogels. Hydrogels of various stiffnesses were ordered from Marigen Life Technologies. Gels were coated for $1 \mathrm{~h}$ at $37^{\circ} \mathrm{C}$ with fibronectin-collagen I mix $\left(2.5 \mu \mathrm{g} \mathrm{ml}^{-1}\right.$ each) before use.

Nuclear fractionation. In order to separate the nucleus from the cytoplasmic fraction, cells were scraped in $1 \mathrm{ml}$ PBS (from $6 \mathrm{~cm}$ dishes) and collected by centrifugation. The cell pellet was suspended in $200 \mu \mathrm{l}$ of cold PBS containing $0.1 \%$ NP-40 followed by $30 \mathrm{~s}$ of centrifugation at $10,000 \mathrm{~g}$ and $4{ }^{\circ} \mathrm{C}$. The supernatant was collected and marked as cytoplasm (C). The pellet was resuspended into $1 \mathrm{ml}$ of cold PBS- $0.1 \%$ NP-40 buffer, and centrifugation was conducted as described earlier. The supernatant was discarded and the nuclear pellet was suspended in $20 \mu \mathrm{l}$ of lysis buffer. The same protein amount was then loaded on 4-20\% SDS-PAGE gel. Lamin $\mathrm{A} / \mathrm{C}$ was used as a nuclear and GAPDH as a cytoplasmic fraction marker.

Transfections. siRNA transfections were performed using Lipofectamine RNAiMAX transfection reagent according to the manufacturer's instructions. Allstars negative control siRNA from Qiagen (siControl_1) and non-targeting siRNA from Dharmacon (siControl_2) were used as controls. Three independent siRNAs were used to silence JMJD1a: JMJD1a_1 custom siRNA (sense: $5^{\prime}$-GUCUA UGUGGGAAUUCCCA-3', antisense: 5'-UGGGAAUUCCCACAUAGAC- $3^{\prime}$ ) was ordered based on previous publication ${ }^{27}$ from Sigma. Dharmacon JMJDla siRNA (JMJD1a_2) was used to validate the results. Third siRNA (siJMJD1a_3; sense $5^{\prime}$-GCAAUUGGCUUGUGGUUACUU- ${ }^{\prime}$ and antisense $5^{\prime}$-GUAACCACAAGCC AAUUGCUU- $3^{\prime}$ ) was ordered based on previous publication ${ }^{57}$ from Sigma. eGFP-JMJD1a (EX-T3698-M29) constructs were ordered from GeneCopoeia. pQCXIH-Myc-YAP (Addgene plasmid \#33091) and pQCXIH-Myc-YAP-5SA (ref. 44; Addgene plasmid \#33093) were gifts from Kunliang Guan and constitutively active Src pLNCX chick src Y527F (Addgene plasmid \# 13660) was a gift from Joan Brugge.

Proliferation assays using YAP overexpression. Overall, 100,000 MDA-MB-231 cells were plated on CDM or plastic overnight. Next day, cells were transfected with 
control, YAP or YAP-5SA plasmid; the plate was placed in Incucyte-ZOOM live-cell incubator and proliferation was monitored over several days. Alternatively, MDA-MB-231 cells were transfected with control or JMJD1a siRNA for $48 \mathrm{~h}$. siControl and siJMJDla cells were trypsinized, and equal amount of cells were plated on 24-well plates. Next day, the cells were transfected with control, wt-YAP or YAP-5SA, and proliferation was monitored in Incucyte-ZOOM over several days.

Matrix solubilization. Matrices were prepared as described above. In order to solubilize matrix proteins, the matrices were incubated in buffer containing $5 \mathrm{M}$ guanidine, $10 \mathrm{mM}$ dithiothreitol (DTT) and $5 \mathrm{mM}$ phenylmethanesulfonylfluoride (PMSF) and incubated for $10 \mathrm{~min}$ at RT. Dissolved matrices were replated on 24-well plates by $1 \mathrm{~h}$ incubation at $37^{\circ} \mathrm{C}$. The wells were washed extensively with PBS.

Coculture experiments. Overall, 5,000 SCC cells were plated on 24-well plates. Next day, 10,000 fibroblasts were plated inside the Transwell inserts $(3 \mu \mathrm{m}$ pore polycarbonate membrane, Corning). In addition, the amount of SCC cells (day 0 time point) was measured with WST-1 staining before adding the fibroblasts. The WST-1 reagent (Roche) was diluted 1:10 in full medium, added to the cells and incubated at $37^{\circ} \mathrm{C}$ for $30 \mathrm{~min}$. Absorbance at $450 \mathrm{~nm}$ was measured using the Multiscan Ascent plate reader (Thermo Scientific).

Tumour growth assay in eggs. Fertilized chicken eggs were incubated as previously described in ref. 57 . Shortly, the eggs were washed and the development was started by placing the eggs in $37^{\circ} \mathrm{C}$ incubator. On day 3 of development, a small hole was made in the eggshell to drop the CAM. On developmental day 10, a plastic ring was placed on the CAM and one million either control or JMJDla siRNA-transfected MDA-MB-231 cells were implanted inside the ring in $20 \mu \mathrm{l}$ of $50 \%$ Matrigel. After 3 days, tumours were imaged and dissected. Tumours were fixed with $4 \%$ PFA overnight and processed for paraffin sections.

Orthotopic breast tumour growth assay. MDA-MB-231 cells were transfected with control and JMJDla siRNA for 3 days (first transfections) and $24 \mathrm{~h}$ (second transfection) before the injection. Two million siControl or siJMJD1a were injected into the abdominal fat pads of 6-week-old virgin female nude mice (NOD.SCID (from Envigo)). Cells were injected into both sides so that siControl cells were on one side and siJMJD1a on the other side. Altogether, 19 mice were used. Ten mice had control tumours on left (and siJMJDla tumours on right) and nine had control cells on the right (and siJMJD1a tumours on the left). Tumour growth was measured and tumours were collected 8 days after the injection. All animal experiments were ethically assessed and authorized by the National Animal Experiment Board (ESAVI/7522/04.10.03/ 2012) and in accordance with The Finnish Act on Animal Experimentation.

\section{Clinical samples and immunohistochemistry. Normal breast tissue and} cancerous primary tumour tissues with their lymph node metastasis counterparts were collected from archives of the Department of Pathology, Helsinki University Central Hospital. Haematoxylin-eosin-stained tissue sections were reviewed, and tissues representative for primary tumour and corresponding nodal metastases or healthy breast tissues were selected for the study. A clinical breast cancer series from the FinHer study was collected from patients with axillary node-positive or high-risk node-negative tumours and from those who had undergone a breast surgery with auxiliary node dissection or sentinel node biopsy for invasive breast carcinoma as described in details elsewhere ${ }^{45}$. Tissue microarrays were constructed from tumourrepresentative tissue regions. Patients, who participated in the FinHer study, provided written informed consent for research use of tumour tissue. An ethics committee at the Helsinki University Central Hospital has approved the study (HUS 106/13/03/02/ 2015). Cancerous primary tumour tissues from patients with HNSCC were collected from archives of the Department of Pathology, Turku University Central Hospital.

Standard immunohistochemistry techniques were applied to detect JMJD1a, YAP and $\alpha$-SMA expression in tissue sections. Briefly, endogenous peroxidase activity was blocked in $1 \%$ hydrogen peroxidase in deparaffinized tissues, and heat-induced epitope retrieval was performed in sodium citrate by using 2100 Antigen Retriever (Aptum Biologics Ltd., UK). Primary antibodies were diluted in Normal antibody Diluent (Immunologic, the Netherlands) and incubated at $4{ }^{\circ} \mathrm{C}$ overnight for JMJDla and YAP (12835-1-AP, Proteintech, dilution 1:100; 63.7, Santa Cruz Biotechnology, dilution 1:250, respectively) or $1 \mathrm{~h}$ at RT for $\alpha$-SMA (clone 1A4, Dako, Denmark, dilution 1:200). Binding of the primary antibody was detected and visualized by using N-Histofine Simple Stain MAX PO Kits (Nichirei Biosciences Inc., Tokyo, Japan) and 3,3'-diaminobenzidine (ImmPACT DAB, Vector Laboratories, Burlingame, CA, USA) following the manufacturers' recommendations. Cytoplasmic JMJDla and YAP expression was classified into four groups (negative, low, intermediate and high) depending on how strong the staining intensity was detected on the majority of tumour cells, whereas nuclear staining was considered as positive whenever more than $10 \%$ of tumour cell nuclei showed protein expression.

Frequency tables were analysed using the $\chi^{2}$-test. Distant disease survival was calculated from the date of randomization to the date of breast cancer recurrence outside of the locoregional region or to the date of death, whenever death preceded distant recurrence. Overall survival was calculated from the date of randomization to the date of death. Survival was analysed using the Kaplan-Meier method, and survival between groups was compared with the log-rank test. All $P$ values are two-sided.

Statistical analyses. The GraphPad programme was used for all statistical analyses. Student's $t$-test (paired, two-tailed) was used in most cases. Nonparametric Mann-Whitney test was used when two unpaired groups were compared and normality could not be tested (because of a too small data set $(n<8)$ ). Unpaired $t$-test was used when normality could be tested. Normality was tested by D’Agostino and Pearson omnibus normality test.

Adhesion assay. We have used an xCELLigence Real-Time Cell Analysis instrument in order to measure cell adhesion on solubilized matrices. Briefly, CDMs derived from NFs or from CAFs were solubilized with Guanidine buffer (5 M guanidine, $10 \mathrm{mM}$ DTT and $5 \mathrm{mM}$ PMSF) for $15 \mathrm{~min}$ at $37^{\circ} \mathrm{C}$. In all, $50 \mathrm{\mu g} \mathrm{ml}^{-1}$ of each solubilized CDM or $0.2 \%$ BSA was plated on of 96 -well E-plates, incubated for $1 \mathrm{~h}$ at $37^{\circ} \mathrm{C}$, washed with PBS and blocked with $0.2 \%$ BSA in PBS for $30 \mathrm{~min}$ at $37^{\circ} \mathrm{C}$. Overall, 100,000 MDA-MB-231 cells were added to each well right before the measurement was started. The cell index was measured every $3 \mathrm{~min}$ for $2 \mathrm{~h}$ and every $10 \mathrm{~min}$ for another $2 \mathrm{~h}$. Student's $t$-test (paired, two-tailed) was used to test for statistical significance.

Data availability. The mRNAseq and Illumina data have been deposited in the Gene Expression Omnibus (GEO) under the accession codes GSE83314 and GSE83366, respectively. All relevant data are either contained in the paper or supplementary files or are available from the authors.

\section{References}

1. Bhowmick, N. A. \& Moses, H. L. Tumor-stroma interactions. Curr. Opin. Genet. Dev. 15, 97-101 (2005).

2. Tilghman, R. W. et al. Matrix rigidity regulates cancer cell growth and cellular phenotype. PLoS ONE 5, e12905 (2010).

3. Yamaguchi, H. \& Sakai, R. Direct interaction between carcinoma cells and cancer associated fibroblasts for the regulation of cancer invasion. Cancers (Basel) 7, 2054-2062 (2015).

4. Baker, E. L., Bonnecaze, R. T. \& Zaman, M. H. Extracellular matrix stiffness and architecture govern intracellular rheology in cancer. Biophys. J. 97, 1013-1021 (2009).

5. Ulrich, T. A., de Juan Pardo, E. M. \& Kumar, S. The mechanical rigidity of the extracellular matrix regulates the structure, motility, and proliferation of glioma cells. Cancer Res. 69, 4167-4174 (2009).

6. Hanahan, D. \& Weinberg, R. A. Hallmarks of cancer: the next generation. Cell 144, 646-674 (2011).

7. Fujii, M., Mori, H., Kato, K. \& Takahashi, M. Cytochemical studies of LDH isoenzymes in experimental bladder tumors. J. Urol. 128, 1349-1352 (1982).

8. Hayashi, N., Cunha, G. R. \& Wong, Y. C. Influence of male genital tract mesenchymes on differentiation of Dunning prostatic adenocarcinoma. Cancer Res. 50, 4747-4754 (1990).

9. Weaver, V. M. et al. Reversion of the malignant phenotype of human breast cells in three-dimensional culture and in vivo by integrin blocking antibodies. J. Cell Biol. 137, 231-245 (1997).

10. Flaberg, E. et al. High-throughput live-cell imaging reveals differential inhibition of tumor cell proliferation by human fibroblasts. Int. J. Cancer 128, 2793-2802 (2011).

11. Sadlonova, A. et al. Human breast fibroblasts inhibit growth of the MCF10AT xenograft model of proliferative breast disease. Am. J. Pathol. 170, 1064-1076 (2007).

12. Gaggioli, C. et al. Fibroblast-led collective invasion of carcinoma cells with differing roles for RhoGTPases in leading and following cells. Nat. Cell Biol. 9, 1392-1400 (2007)

13. DuFort, C. C., Paszek, M. J. \& Weaver, V. M. Balancing forces: architectural control of mechanotransduction. Nat. Rev. Mol. Cell Biol. 12, 308-319 (2011)

14. Charras, G. \& Sahai, E. Physical influences of the extracellular environment on cell migration. Nat. Rev. Mol. Cell Biol. 15, 813-824 (2014).

15. Hirata, E. et al. Intravital imaging reveals how BRAF inhibition generates drugtolerant microenvironments with high integrin beta1/FAK signaling. Cancer Cell. 27, 574-588 (2015)

16. Dupont, S. et al. Role of YAP/TAZ in mechanotransduction. Nature 474, 179-183 (2011)

17. Okada, Y., Scott, G., Ray, M. K., Mishina, Y. \& Zhang, Y. Histone demethylase JHDM2A is critical for Tnp1 and Prm1 transcription and spermatogenesis. Nature 450, 119-123 (2007).

18. Tateishi, K., Okada, Y., Kallin, E. M. \& Zhang, Y. Role of Jhdm2a in regulating metabolic gene expression and obesity resistance. Nature 458, 757-761 (2009).

19. Yamane, K. et al. JHDM2A, a JmjC-containing H3K9 demethylase, facilitates transcription activation by androgen receptor. Cell 125, 483-495 (2006).

20. Cukierman, E., Pankov, R., Stevens, D. R. \& Yamada, K. M. Taking cell-matrix adhesions to the third dimension. Science 294, 1708-1712 (2001). 
21. Dahl, K. N., Ribeiro, A. J. \& Lammerding, J. Nuclear shape, mechanics, and mechanotransduction. Circ. Res. 102, 1307-1318 (2008).

22. Jaalouk, D. E. \& Lammerding, J. Mechanotransduction gone awry. Nat. Rev. Mol. Cell Biol. 10, 63-73 (2009).

23. Osmanagic-Myers, S., Dechat, T. \& Foisner, R. Lamins at the crossroads of mechanosignaling. Genes Dev. 29, 225-237 (2015).

24. Wang, N., Tytell, J. D. \& Ingber, D. E. Mechanotransduction at a distance: mechanically coupling the extracellular matrix with the nucleus. Nat. Rev. Mol. Cell Biol. 10, 75-82 (2009).

25. Lelievre, S. A. Contributions of extracellular matrix signaling and tissue architecture to nuclear mechanisms and spatial organization of gene expression control. Biochim. Biophys. Acta 1790, 925-935 (2009).

26. Kutys, M. L., Doyle, A. D. \& Yamada, K. M. Regulation of cell adhesion and migration by cell-derived matrices. Exp. Cell Res. 319, 2434-2439 (2013).

27. Cho, H. S. et al. The JmjC domain-containing histone demethylase KDM3A is a positive regulator of the G1/S transition in cancer cells via transcriptional regulation of the HOXA1 gene. Int. J. Cancer 131, E179-E189 (2012).

28. Krieg, A. J. et al. Regulation of the histone demethylase JMJD1A by hypoxiainducible factor 1 alpha enhances hypoxic gene expression and tumor growth. Mol. Cell Biol. 30, 344-353 (2010).

29. Zhao, W. et al. Jmjd3 inhibits reprogramming by upregulating expression of INK4a/Arf and targeting PHF20 for ubiquitination. Cell 152, 1037-1050 (2013).

30. Calvo, F. et al. Mechanotransduction and YAP-dependent matrix remodelling is required for the generation and maintenance of cancer-associated fibroblasts. Nat. Cell Biol. 15, 637-646 (2013).

31. Naba, A. et al. The matrisome: in silico definition and in vivo characterization by proteomics of normal and tumor extracellular matrices. Mol. Cell Proteomics 11, M111.014647 (2012).

32. Kalluri, R. \& Zeisberg, M. Fibroblasts in cancer. Nat. Rev. Cancer 6, 392-401 (2006).

33. Quante, M. et al. Bone marrow-derived myofibroblasts contribute to the mesenchymal stem cell niche and promote tumor growth. Cancer Cell. 19, 257-272 (2011)

34. Paszek, M. J. et al. Tensional homeostasis and the malignant phenotype. Cancer Cell 8, 241-254 (2005).

35. Kim, K. Y. et al. Kaposi's sarcoma-associated herpesvirus (KSHV) latencyassociated nuclear antigen regulates the KSHV epigenome by association with the histone demethylase KDM3A. J. Virol. 87, 6782-6793 (2013).

36. Aragona, M. et al. A mechanical checkpoint controls multicellular growth through YAP/TAZ regulation by actin-processing factors. Cell 154, 1047-1059 (2013).

37. Halder, G., Dupont, S. \& Piccolo, S. Transduction of mechanical and cytoskeletal cues by YAP and TAZ. Nat. Rev. Mol. Cell Biol. 13, 591-600 (2012).

38. Lee-Thedieck, C. \& Spat, J. P. Biophysical regulation of hematopoietic stem cells. Biomater. Sci. 2, 1548-1561 (2014).

39. Dupont, S. Role of YAP/TAZ in cell-matrix adhesion-mediated signalling and mechanotransduction. Exp. Cell Res. 343, 42-53 (2015).

40. Tang, Y. et al. MT1-MMP-dependent control of skeletal stem cell commitment via a betal-integrin/YAP/TAZ signaling axis. Dev. Cell 25, 402-416 (2013)

41. Das, A., Fischer, R. S., Pan, D. \& Waterman, C. M. YAP nuclear localization in the absence of cell-cell contact is mediated by a filamentous actin-dependent, myosin II- and phospho-YAP-independent pathway during extracellular matrix mechanosensing. J. Biol. Chem. 291, 6096-6110 (2016).

42. Kim, N. G. \& Gumbiner, B. M. Adhesion to fibronectin regulates Hippo signaling via the FAK-Src-PI3K pathway. J. Cell Biol. 210, 503-515 (2015).

43. Zhao, B. et al. Cell detachment activates the Hippo pathway via cytoskeleton reorganization to induce anoikis. Genes Dev. 26, 54-68 (2012).

44. Zhao, B. et al. Inactivation of YAP oncoprotein by the Hippo pathway is involved in cell contact inhibition and tissue growth control. Genes Dev. 21, 2747-2761 (2007)

45. Joensuu, H. et al. Adjuvant docetaxel or vinorelbine with or without trastuzumab for breast cancer. N. Engl. J. Med. 354, 809-820 (2006).

46. Pickup, M. W., Mouw, J. K. \& Weaver, V. M. The extracellular matrix modulates the hallmarks of cancer. EMBO Rep. 15, 1243-1253 (2014).

47. Conklin, M. W. et al. Aligned collagen is a prognostic signature for survival in human breast carcinoma. Am. J. Pathol. 178, 1221-1232 (2011).

48. Klein, G. Toward a genetics of cancer resistance. Proc. Natl Acad. Sci. USA 106, 859-863 (2009).

49. Chen, H., Kluz, T., Zhang, R. \& Costa, M. Hypoxia and nickel inhibit histone demethylase JMJD1A and repress Spry2 expression in human bronchial epithelial BEAS-2B cells. Carcinogenesis 31, 2136-2144 (2010).
50. Parrish, J. K., Sechler, M., Winn, R. A. \& Jedlicka, P. The histone demethylase KDM3A is a microRNA-22-regulated tumor promoter in Ewing Sarcoma. Oncogene 34, 257-262 (2015).

51. Uemura, M. et al. Jumonji domain containing 1A is a novel prognostic marker for colorectal cancer: in vivo identification from hypoxic tumor cells. Clin. Cancer Res. 16, 4636-4646 (2010).

52. Park, S. J. et al. The histone demethylase JMJD1A regulates adrenomedullinmediated cell proliferation in hepatocellular carcinoma under hypoxia. Biochem. Biophys. Res. Commun. 434, 722-727 (2013).

53. Piccolo, S., Dupont, S. \& Cordenonsi, M. The biology of YAP/TAZ: hippo signaling and beyond. Physiol. Rev. 94, 1287-1312 (2014).

54. Pobbati, A. V. \& Hong, W. Emerging roles of TEAD transcription factors and its coactivators in cancers. Cancer Biol. Ther. 14, 390-398 (2013).

55. Lansford, C. D. et al. in Human Cell Culture, Vol II, Cancer Cell Lines Part 2. (eds Masters, J. W. R. \& Palsson, B.) 185-255 (Kluwer Academic Press, 1999).

56. Hertz, H. Uber den Kontakt elastischer Korper. Mathematik 92, 156-156 (1881).

57. Beyer, S., Kristensen, M. M., Jensen, K. S., Johansen, J. V. \& Staller, P. The histone demethylases JMJD1A and JMJD2B are transcriptional targets of hypoxia-inducible factor HIF. J Biol Chem. 283, 36542-36552 (2008).

58. Hagedorn, M. et al. Domain swapping in a $\mathrm{COOH}$-terminal fragment of platelet factor 4 generates potent angiogenesis inhibitors. Cancer Res. 62, 6884-6890 (2002).

\section{Acknowledgements}

We thank P. Laasola, J. Siivonen, S. Vuorikoski, O. Levälampi and M. Lindman for excellent technical assistance. H. Hamidi is acknowledged for scientific writing and editing the manuscript. FMSC and CIC core facilities at the Turku Centre for Biotechnology are acknowledged for their help with the gene expression and imaging. Helsinki Electron microscopy unit at the Institute of Biotechnology, University of Helsinki, are acknowledged for the electron microscopy. This study has been supported by the Academy of Finland, an ERC Starting and Consolidator Grant 615258, the Sigrid Juselius Foundation, the Finnish Cancer Organisation and the Finnish Microarray and Sequencing Centre, University of Turku and Åbo Akademi University and Biocenter Finland. J.I. has been supported by Labex CelTisPhyBio fellowship. R.K. has been supported by the Orion-Farmos Foundation, the Finnish Cancer Organization, the K. Albin Johansson Foundation and the Instrumentarium Foundation and the Turku Doctoral Programme of Biomedical Sciences. T.B. was supported by the Deutsche Forschungsgemeinschaft (DFG), Cells-in-Motion Cluster of Excellence (EXC 1003-CiM), University of Münster, Germany.

\section{Author contributions}

R.K. designed, carried out and analysed the majority of the experiments with help from A.M., M.G., N.D.F., J.I. and M.S. J.I. and R.K. wrote the manuscript. T.B. analysed the AFM data, L.E. analysed the gene expression data and E.J. performed SEM experiments. J.W., S.V., P.-L.K.-L., H.S., H.J and R.G. provided the clinical samples, cell lines and the tissue sample analyses. J.I. supervised the study.

\section{Additional information}

Supplementary Information accompanies this paper at http://www.nature.com/ naturecommunications

Competing financial interests: The authors declare no competing financial interests.

Reprints and permission information is available online at http://npg.nature.com/ reprintsandpermissions/

How to cite this article: Kaukonen, R. et al. Normal stroma suppresses cancer cell proliferation via mechanosensitive regulation of JMJDla-mediated transcription. Nat. Commun. 7:12237 doi: 10.1038/ncomms12237 (2016)

This work is licensed under a Creative Commons Attribution 4.0 International License. The images or other third party material in this article are included in the article's Creative Commons license, unless indicated otherwise in the credit line; if the material is not included under the Creative Commons license, users will need to obtain permission from the license holder to reproduce the material To view a copy of this license, visit http://creativecommons.org/licenses/by/4.0/

C) The Author(s) 2016 